\title{
Effects of kinetic processes in shaping Io's global plasma environment: A 3D hybrid model
}

\author{
Alexander S. Lipatov ${ }^{1,2}$, and Michael R. Combi ${ }^{1}$ \\ ${ }^{1}$ Space Physics Research Laboratory, Department of Atmospheric, Oceanic and Space \\ Sciences, The University of Michigan, Ann Arbor, MI 48109-2143 \\ 2 also Dialogue-Science, Computing Center Russian Academy of Sciences, Vavilova St. \\ 40, 119991 Moscow, Russia
}

Number of pages - 42

Number of figures - 20

Number of tables - 1 
Corresponding author: A. S. Lipatov, Space Physics Research Laboratory, Department of Atmospheric, Oceanic and Space Sciences, The University of Michigan, Ann Arbor, MI 48109-2143, Phone: (734) 763-6246, FAX: (734) 647-3083 email: alipatov@engin.umich.edu

Short title: IO'S PLASMA ENVIRONMENT 
Abstract. The global dynamics of the ionized and neutral components in the environment of Io plays an important role in the interaction of Jupiter's corotating magnetospheric plasma with Io. The stationary simulation of this problem was done in the MHD and the electrodynamics approaches. One of the main significant results from the simplified two-fluid model simulations (Saur et al., 2002) was a production of the structure of the double-peak in the magnetic field signature of the I0 flyby that could not be explained by standard MHD models. In this paper, we develop a method of kinetic ion simulation. This method employs the fluid description for electrons and neutrals whereas for ions multilevel, drift-kinetic and particle, approaches are used. We also take into account charge-exchange and photoionization processes. Our model provides much more accurate description for ion dynamics and allows us to take into account the realistic anisotropic ion distribution that cannot be done in fluid simulations. The first results of such simulation of the dynamics of ions in the Io's environment are discussed in this paper.

KEYWORDS: Io; Jupiter; Magnetosphere; Satellites; Atmospheres. 


\section{Introduction}

The global interaction of the Jovian plasma torus with the Io is a fundamental problem in magnetospheric physics. It requires the solution of a highly nonlinear coupled set of integro-MHD/kinetic-Boltzmann equations which describe the dynamics of Jupiter's corotating magnetospheric plasma, pickup ions and the ionosphere together with the atoms from Io's environment. To leading order, the plasma and neutral atoms are coupled by resonant charge exchange, although other coupling processes are present. The characteristic scale of the ionized components is determined usually by the typical ion gyroradius, which for Io is much less than characteristic global magnetospheric scales of interest. By contrast, the mean free path of neutral particles and the typical ion gyroradius are comparable to characteristic exospheric scales such as the thickness of the exosphere and atmosphere, the distances separating the possible magnetic barriers and the surface of Io, etc. Consequently, the Knudsen number $K n=\lambda / L_{\text {exo }}(\lambda$, the mean free path of neutral particles and $L_{\text {exo }}$, a characteristic exospheric scale), which is a measure of the distribution relaxation distance, satisfies $K n \approx 1$. Thus it is difficult to assume that the distribution of the neutral atoms and ions can relax to a Maxwellian distribution, and one needs ideally to solve a Boltzmann equation for the neutral and ionized components in which charge exchange and photoionization processes are included.

Several approaches for including the neutral component and pickup ions selfconsistently in models describing the interaction of plasma torus with Io have been formulated. Early theoretical work was often done either in the context of a "thin" 
atmosphere (e.g., see Cloutier et al., 1978) indicative of the surface temperature ( $130 \mathrm{~K})$, or "thick" extended neutral atmosphere (e.g., see Goertz, 1980) more indicative of volcanic temperatures $(1000 \mathrm{~K})$. Subsequent evidence (see the review by Lellouch, 1996) seems to indicate a mixed picture of the global atmosphere, which has a large extended corona like a thick atmosphere, but appears to be dominated by local major injection of hot (high speed) gas/dust plumes to high altitudes but only near active volcanic vents. Therefore, although the atmosphere is probably only locally thick, it still has a large extended neutral corona which might provide a sufficient source of impact ionization and photoionization to explain the plasma torus.

Southwood et al. (1980) examined data from several Voyager instruments and examined the possible role of an intrinsic magnetic field for Io as a way to retain a robust enough ionosphere, which could provide enough conductivity for completing the Io-Jupiter current circuit. Neubauer (1980) presented an analytical model of the Alfvén standing wave current system which connects current through the ionosphere of Io. Southwood and Dunlop (1984) and Ip (1990) suggested models stating that mass loading effects should result in the formation of a tail-like structure in the wake behind Io; as a consequence of the enhanced plasma density, the magnetic field perturbations are continued into the wake. Thus the field-aligned current is not only generated by Io itself, but also in the wake. Several years after Voyager, 3D numerical studies of the plasma flow past Io were performed using electrodynamic (Wolf-Gladrow et al., 1987), magnetohydrodynamic (Linker et al. 1989; Linker et al. 1991) and resistive magnetohydrodynamic (Kopp, 1996; Kopp, Birk and Otto, 1998) approaches. 
There have been recent efforts to improve and extend the pre-Galileo simulations both in terms of the MHD (Combi et al., 1998; Linker et al., 1998; Kabin et al., 2001) and the electrodynamic (Saur et al., 1999) approaches. These two approaches are distinguished by the physical assumptions which they each do and do not (or in some cases, can and cannot) include. However, MHD cannot, at least yet, include the effects of realistic conductivities (Hall and Pederson) or charge separation effects which are likely to be important very close to Io where the neutral densities are large and electric potential can introduce non-symmetric flow around body. They either include constant artificial conductivity (Linker et., 1998) or assume perfect conductivity (Combi et al., 1998), however comparisons of the sets of published results do not indicate that this choice has any important consequence.

The non-magnetic models produce magnetic field perturbations that are similar to the Galileo measurements, but none are quite as deep or as broad, and none have the reversal of the perturbation (the double-peaked structure) in the center of the wake. The magnetized models of Linker et al. (1998) produced a broad and deep perturbation, but not the self-reversal at the center of the wake (the double-peak or bite-out). Thus, the observational data, e.g., density and magnetic field profile along the Galileo trajectory cannot be fully explained by MHD models. But the most recent evidence from all the Galileo flyby data is that Io does not possess any substantial internal magnetic field of its own (Kivelson et al., 2001).

In the electrodynamical model of Saur et al. (2002), they added an extra ionization source, based on the high-energy bidirectional electrons observed by Williams et al. 
$(1996,1999)$ and Frank and Paterson (1999). These high-energy electrons were included as an energy source in addition to electron impact ionization by the thermal electrons and photoionization to create a dense plasma wake as is observed. Simulations without these extra bidirectional electrons show a nearly empty wake Saur et al. (1999). They also concluded that the electrodynamic part of Io's interaction is best described as an ionosphere-like interaction rather than a comet-like interaction (Saur et al., 2003). Saur et al. (2002) demonstrated first that the diamagnetic and inertia currents are responsible for formation of the double-peak magnetic profile along the I0 trajectory. They received the magnetic field profile that has an oscillating structure and the maximum in the magnetic field profile is to some extend was a narrower than in observation for standard model of the atmosphere (Fig. 11, Saur et al., 2002). In a case of longitudinally symmetric atmosphere they received a strong double-pick structure but the spatial scale of this structure was a twice smaller than in observation (Fig. 14, Saur et al., 2002) and the space scale of these peaks are much smaller than in observation.

In this paper, we describe a new approach to solving the time-dependent Boltzmann equation together with a hybrid plasma (ion kinetic) model in three spatial dimensions using a prescribed atmosphere model for Io. A Boltzmann simulation is applied to model charge exchange between (incoming and pickup) ions and immobile atmosphere. Several simulations are run, and the results described. We show for the first time the predicted distribution for ions and the electromagnetic field throughout Io's environment. The results of such kinetic simulations are compared with those obtained from a related 
MHD model and observational data. We also found the best parameters for our model that describe satisfactory the main behavior of the observational data at least on the qualitative level.

\section{Formulation of the Problem and Mathematical Model}

\subsection{Simulation Model}

To study the interaction of the plasma torus with the ionized and neutral components of Io's environment we use quasineutral hybrid models for ions and electrons. The interaction of the ions with atmosphere is dominated by charge exchange. The atmosphere is considered to be an immobile component in this paper. The general scheme of the global interaction of the plasma torus with Io and the Galileo I0 trajectory is given in Fig. 1. The Galileo I0 flyby occurred nearly in the equator plane of Io and perpendicular to the direction of the plasma wake defined by the corotating plasma flow past Io. The spacecraft trajectory passed approximately $900 \mathrm{~km}$ down-stream of Io (in the sense of the plasma torus flow). In our coordinate system the $Z$ axis is parallel to $U_{0}$ (corotational plasma velocity), $Y$ is aligned with the spin axis, and $\mathbf{X}=\mathbf{Y} \times \mathbf{Z}$. The relation between our coordinate system and IphiO $\left(X^{*}, Y^{*}, Z^{*}\right)$ system (see Fig. 3 from Kivelson et al., 2001) is the following: $X$ is parallel to $Y^{*}, Y$ is aligned with $Z^{*}$, and $Z$ is parallel to $X^{*}$.

In the hybrid simulations described here, the dynamics of upstream ions and implanted ions is described in a kinetic approach, while the dynamics of the electrons is described in a hydrodynamical approximation. 
The single particle ion distribution function $f_{s}(t, \mathbf{x}, \mathbf{v})$ has to fulfill the Vlasov equation

$$
\frac{\partial}{\partial t} f_{s}+\mathbf{v} \frac{\partial}{\partial \mathbf{x}} f_{s}+\frac{\mathbf{F}}{M_{s}} \frac{\partial}{\partial \mathbf{v}} f_{s}=0
$$

where $\mathbf{F}$ symbolizes all forces acting on the ions.

The single ion particle motion is described by the equations

$$
\frac{d \mathbf{r}_{s, l}}{d t}=\mathbf{v}_{s, l} ; \quad \frac{d \mathbf{v}_{s, l}}{d t}=\frac{Z_{s} e}{M_{s}}\left(\mathbf{E}^{*}+\frac{\mathbf{v}_{s, l} \times \mathbf{B}}{c}\right), \quad \mathbf{E}^{*}=\mathbf{E}-\sigma_{e f f}^{-1} \mathbf{J}
$$

Here $Z_{s}$ and $M_{s}$ denote the charge state and the mass of the ions, the subscript $s$ denotes the ion species ( $s=1$ for incoming ions and $s=2$ for pickup ions) and the index $l$ is the particle index. $\sigma_{e f f}$ is an effective conductivity that may include Coulomb collisions and collisions due to particle-wave interaction, in the sense of a standard resistive Ohm's law. Note that the resistivity used in Eq. (2) must depend on individual velocities of ions and electrons. However, we use the effective resistivity and an effective electric field $E^{*}$ in Eq. (2) to satisfy momentum conservation for the plasma system (Zueva et al., 1975).

In the nonradiative limit Ampère's law is given by

$$
\frac{4 \pi}{c} \mathbf{J}=\nabla \times \mathbf{B}
$$

and the induction equation (Faraday's law) by

$$
\frac{1}{c} \frac{\partial \mathbf{B}}{\partial t}+\nabla \times \mathbf{E}=0
$$

The total current is given by

$$
\mathbf{J}=\mathbf{J}_{\mathrm{e}}+\mathbf{J}_{\mathrm{i}} ; \quad \mathbf{J}_{\mathrm{i}}=\sum_{s=1}^{2} Z_{s} e n_{s} \mathbf{U}_{s}
$$


here $\mathbf{U}_{s}$ is the bulk velocity of ions of the type $s$.

We further assume quasi-neutrality

$$
n_{\mathrm{e}}=\sum_{s=1}^{2} Z_{s} n_{s}
$$

For massless electrons the equation of motion of the electron fluid takes the form of standard generalized Ohm's law (e.g. Braginskii, 1965):

$$
\mathbf{E}=\frac{1}{e n_{\mathrm{e}} c}\left(\mathbf{J}_{\mathrm{e}} \times \mathbf{B}\right)-\frac{1}{e n_{\mathrm{e}}} \nabla p_{\mathrm{e}}+\frac{1}{e n_{\mathrm{e}}} \mathbf{R}_{\mathrm{e}}
$$

where $p_{\mathrm{e}}=n m_{\mathrm{e}}\left\langle v_{\mathrm{e}}^{\prime 2}\right\rangle / 3=n_{\mathrm{e}} k_{B} T_{\mathrm{e}}$, and $v_{\mathrm{e}}^{\prime}$ are the scalar electron pressure and the thermal velocity of electrons, and the electron current is estimated from Eq. ( 5).

The term including $\mathbf{R}_{\mathrm{e}}$, symbolizes the mean momentum change of the electrons due to their collisions with the ions, which can be parametrized in terms of the collision frequency $\nu_{e s}$ between electrons and ions of the species $s$ as

$$
\mathbf{R}_{\mathrm{e}}=-n_{\mathrm{e}} m_{\mathrm{e}} \nu_{\mathrm{es}}\left(\mathbf{U}_{\mathrm{e}}-\mathbf{U}_{\mathrm{s}}\right)
$$

Since we suppose that electron heating due to collisions with ions is very small, the electron fluid is considered adiabatic:

$$
p_{\mathrm{e}} \propto n_{\mathrm{e}}^{5 / 3}
$$

The ion kinetic approach allows us to take into account the effects of anisotropy of ion pressure, the correct mass loading processes, a penetration of ions across the ionosphere, and the asymmetry of plasma flow around the Io. Remember that the fluid models which account only for the scalar ion pressure may result in an extra-effusion of the pickup ions along the Alfvén wing. 
Charge exchange may be included in a hybrid model by a simple procedure (Lipatov et al., 1998; Lipatov, 2002). The total loss rate in $\mathbf{s}^{-1}$ for the ionized component has the form

$$
\beta_{\mathrm{ex}}(\mathbf{x}, \mathbf{v}, t)=\int f_{a}\left(\mathbf{x}, \mathbf{v}_{a}, t\right) V_{r e l, a} \sigma_{e x}\left(V_{r e l, a}\right) d^{3} \mathbf{v}_{a}
$$

where the velocity of ions relative to an atom with velocity $\mathbf{v}_{a}$ is $V_{\text {rel, } a}=\left|\mathbf{v}-\mathbf{v}_{a}\right|$.

If we suppose that the neutral component has a Maxwellian distribution, then $\beta_{e x}$ may be approximated as (Ripkin and Fahr, 1983)

$$
\beta_{\mathrm{ex}}(\mathbf{x}, \mathbf{v}, t) \simeq n_{a}(\mathbf{x}, t) V_{\text {rel, } \mathrm{a}} \sigma_{\mathrm{ex}}\left(V_{\text {rel }, a}\right)
$$

In this paper we assume that the neutral component of Io's atmosphere has zero bulk and thermal velocities. If one needs to include the nonzero temperature and bulk velocity, the effective average velocity of ions relative to an atom with velocity may be found from the general equation of Ripkin and Fahr (1983).

Let the time interval $t^{*}$ with respect to the charge exchange be a random variable with a distribution function

$$
w_{l}\left(t^{*}\right)=\exp \left[-\int_{t_{0}}^{t^{*}} \beta_{\mathrm{ex}, l} d t\right]
$$

where $l$ is the particle index.

A survival probability against the charge exchange event $w_{e x}$ may be written as follows:

$$
w_{\mathrm{ex}, l}(t)=\exp \left[-\int_{t_{0}}^{t} \beta_{\mathrm{ex}, l} d t\right]
$$

The integration is over the trajectory of the particle with index $l$. At the time of creation (either at the boundary of the calculation box, or at the moment of charge 
exchange), an ionized particle has initial coordinates $\mathbf{x}_{l}\left(t_{0}\right)=\mathbf{x}_{l, 0}, \mathbf{v}_{l}\left(t_{0}\right)=\mathbf{v}_{l, 0}$, a weight $\alpha_{l}\left(t_{0}\right)=\alpha_{l, 0}$, and a survival probability $w_{\mathrm{ex}, l}\left(t_{0}\right)=1$. For each new ion with index $l$, we have to determine the critical probability $w_{e x, l}^{*}$ when charge exchange will occur, and this is done using the relation

$$
w_{\mathrm{ex}, l}^{*}=\xi
$$

where $\xi$ is random number on the interval from 0 to 1 . During the calculation we have to identify those particles for which the probability of survival satisfies the condition

$$
w_{\mathrm{ex}, l} \leq w_{\mathrm{ex}, l}^{*}
$$

If the particle satisfies the condition (15), then we have to exchange the velocity of this ion with the velocity of an atom from the atmosphere of Io. In the present simulations, we do not take the cross section $\sigma$ into account in (10) because of the weak dependence on $\left|v-v_{p}\right|$ (as was done in the work of Malama (1991)). If charge exchange occurs, then a new neutral particle begins its motion with $\mathrm{v}_{l}=0$ and $w_{\mathrm{ex}, l}=1$.

Io's environment model includes 2 sources of pickup ions: the extended coma halo distribution with pickup ion production law

$$
G \propto \nu_{i} n_{\mathrm{atmos}} W_{e x t} r^{-2}
$$

for $1 \times r_{\mathrm{I} o}<r<7 \times r_{\mathrm{Io}}$ and the exosphere distribution close to Io with ion production law

$$
G \propto \nu_{i} n_{\text {atmos }} W_{\text {int }} \exp \left[-\left(r-r_{\mathrm{Io}}\right) / H_{\text {atmos }}\right]
$$

Here $W_{\text {ext }}$ and $W_{\text {int }}$ denote the fraction of the total ion production rate in the halo 
and exosphere $\left(W_{\text {int }}=(100-95) \%\right.$ of the total $)$, respectively. $n_{\text {atmos }}$ denotes the maximum atmosphere density, $\nu_{i}$ is effective ionization rate.

The inner region of the Io's ionosphere is described by immobile ions with the following density distributions:

$$
\begin{gathered}
0.5 n_{\text {iono }} \exp \left(-\left(r-r_{\mathrm{Io}}\right) / H_{\text {eff }}\right), \quad \text { for } \quad r>r_{\mathrm{lo}}, \\
n_{\text {iono }}\left(1-0.5 \exp \left(-\left|r-r_{\mathrm{Io}}\right| / H_{\text {eff }}\right)\right), \quad \text { for } \quad r<r_{\mathrm{Io}},
\end{gathered}
$$

We assume that the incoming flow has a small finite resistivity to suppress the "shot" noise fluctuations. We also take into account the effect of finite conductivity of Io's body so that

$$
\begin{aligned}
& \sigma_{\text {eff }}=\sigma_{\mathrm{up}}, \quad \text { for } \quad r>r_{\mathrm{Io}}, \\
& \sigma_{\text {eff }}=\sigma_{\mathrm{Io}}, \quad \text { for } \quad r \leq r_{\mathrm{Io}},
\end{aligned}
$$

Our code solves equations (1) - (8), (5) - (9), (10) - (15) and (16) - (17).

Initially the computational domain contains only supersonic plasma torus flow with a homogeneous spatial distribution and a Maxwellian velocity distribution; the pickup ions have a weak density and spherical spatial distribution. The magnetic field and electric fields are $\mathbf{B}=\mathbf{B}_{0}$ and $\mathbf{E}=\mathbf{U}_{0} \times \mathbf{B}_{0}$. Inside the Io electromagnetic fields are $\mathbf{E}=0$ and $\mathbf{B}=\mathbf{B}_{0}$. In the cases examined in Figs. 9-16 we choose $\mathbf{B}_{0} / B_{0}=(0,1,0) ; \mathbf{E}_{0} / E_{0}=(1,0,0)$ and in the cases examined in Figs. 2-8, 17-19 we choose $\mathbf{B}_{0} / B_{0}=(0.0394,-0.9854,0.1657)$ and $\mathbf{E}_{\mathbf{0}} / E_{0}=(-0.98,-0.0394,0.0)$.

At $t>0$ we begin to inject the pickup ions with a distribution according to Eqs. (16-17). Far upstream $(z=-D Z / 2)$, the ion flux is assumed to have a Maxwellian 
distribution,

$$
f=n_{\infty}\left(\pi v_{t h}^{2}\right)^{-3 / 2} \exp \left[-\frac{(\mathbf{v}-\mathbf{U})^{2}}{2 v_{t h}^{2}}\right]
$$

where $v_{t h}$ and $\mathrm{U}$ are the thermal and the bulk velocities of the plasma torus flow, respectively.

Far downstream, we adopt a free escape condition for particles and Sommerfeld's radiation condition for the magnetic field. On the side boundaries $(y= \pm D Y / 2$ and $x= \pm D X / 2$ ), periodic boundary conditions are imposed for incoming flow particles and the electromagnetic field. In some cases we also tested the use of the upstream boundary condition for electromagnetic field on the side boundaries. In these situations we also employ a buffer zone with thickness about of $10 \times \Delta x$ where a smoothing procedure provides a transition for electromagnetic fields from the perturbed value to the upstream value on the side boundaries (see e.g. Umeda et al., 2001), effectively allowing Iogenerated Alfvén disturbances to propagate away. The pickup ions come out from the computational domain when they intersect the surfaces $x=5 \times \Delta x, x=D X-5 \times \Delta x$, $y=5 \times \Delta y, y=D Y-5 \times \Delta y, z=5 \times \Delta z, z=D Z-5 \times \Delta z$. Thus the flux of the pickup ions is absent on the side boundaries. At Io's surface the particles may be reflected or absorbed. Note that the position of the Io is $x=0, y=0, z=0$.

The three-dimensional computational domain has the dimensions $D X=20 L$, $D Y=20 L$, and $D Z=10 L$, or $D X=14 L, D Y=14 L$, and $D Z=12 L$, or $D X=10 L$, $D Y=10 L$, and $D Z=8 L$, where $L$ equals the radius of Io, $r_{\text {Io }}=1800 \mathrm{~km}$. We use the meshes of $201 \times 201 \times 101$, or $161 \times 161 \times 121$, or $141 \times 141 \times 121$ grid points, and $2 \times 10^{8}$ 
and $5 \times 10^{7}$ particles for ions and pickup ions, respectively, for a homogeneous mesh computation. The time step $\Delta t$ satisfies the condition $v_{\max } \Delta t \leq \min (\Delta x, \Delta y, \Delta z) / 8$.

The relationship between dimensional $\left(U, E, B, p_{\mathrm{e}}, n, T\right)$ and dimensionless $\left(U^{\prime}\right.$, $\left.E^{\prime}, B^{\prime}, p_{\mathrm{e}}^{\prime}, n^{\prime}, T^{\prime}\right)$ parameters may be expressed via dimensional upstream values as follows:

$$
\begin{gathered}
\mathbf{U}=\mathbf{U}^{\prime} U_{0}, \quad \mathbf{E}=\mathbf{E}^{\prime} B_{0} U_{0} / c, \quad \mathbf{B}=\mathbf{B}^{\prime} B_{0}, \quad p_{\mathrm{e}}=p_{\mathrm{e}^{\prime}} p_{\mathrm{e} 0} \\
n=n^{\prime} n_{0}, \quad T=T^{\prime} M_{\mathrm{i}} U_{0}^{2}
\end{gathered}
$$

whereas the dimensional time and distance may be expressed via the bulk velocity $U_{0}$ and characteristic scale $L$ :

$$
t=t^{\prime} L / U_{0}, \quad x=x^{\prime} L
$$

\subsection{Numerical Method}

We employ a standard particle-in-cell (PIC) method in the case of a homogeneous grid. The time integration of the particle motion equations uses a leapfrog scheme. The time integration of the electromagnetic field equations uses an implicit finite difference scheme (see, e.g., Lipatov (2002)). We use different time steps for particle and field pushing (subcycling). This code was optimized for parallel computation using MPI and OMP.

Since the gyroradii must be resolved, a grid point spacing of less than 1 gyroradius is required in order to avoid numerical dispersion and dissipation. On the other hand, good statistics are required, therefore a sufficiently large number of particles per cell have to be used (i.e. low "shot" noise). We use a homogeneous mesh for the simulations 
presented here.

\section{Results of the Simulation}

To study the interaction of the plasma torus with the ionosphere of Io the following set of Jovian plasma torus and ionosphere parameters were adopted in accordance with observational data (Frank et al., 1996; Kivelson et al., 1996): corotation velocity, $U_{0}=56.8 \mathrm{~km} / \mathrm{s} ;$ plasma density, $n_{0}=3500 \mathrm{~cm}^{-3} ;$ plasma temperature, $92 \mathrm{eV} ;$ mean ion mass, $M_{\mathrm{i}}=22 M_{\mathrm{p}} ;$ ratio of specific heats, $\gamma=5 / 3$; ion and electron betas, $\beta_{\mathrm{i}}=0.039$; $\beta_{\mathrm{e}}=0.0022 ;$ magnetic field, $B=1800 \mathrm{nT}$; Alfvén and sonic Mach numbers $M_{\mathrm{A}}=0.4$ and $M_{\mathrm{S}}=2.2$.

For pickup ions we use the following parameters: total ion production rate, $Q_{\text {ion }}=(0.7-2.) \times 10^{28} \mathrm{~s}^{-1} ;$ mean ion mass, $M_{\mathrm{PI}}=22 M_{\mathrm{p}} ;$ electron exosphere and ionosphere betas, $\beta_{\mathrm{e}, \mathrm{exo}}=0-0.0022, \beta_{\mathrm{e}, \mathrm{iono}}=0-0.0005$; effective cross section for charge exchange, $\sigma_{\text {exch }}=1.5 \times 10^{-15} \mathrm{~cm}^{2}$; effective ionization rate, $\nu_{i}=10^{-5} \mathrm{~s}^{-1}$; atmosphere scale height, $H_{\text {atmos }}=0.06-0.09 \times r_{\mathrm{I} 0}=108 \mathrm{~km}-162 \mathrm{~km}$; maximum value of the density of the atmosphere, $n_{\text {atmos }}=(0.5-10) \times 10^{8} \mathrm{~cm}^{-3}$; effective ionosphere scale height, $H_{e f f}=(0.0001-0.00025) \times r_{\mathrm{Io}}=(0.18-0.45) \mathrm{km}$. Note that in calculation the effective ionosphere scale height is smoothed over the nearest grid cell and its specific values is not crucial. However, for charge exchange between the ions and atoms we use the analytical formula for the density of the atmosphere without any smoothing. The effective dimensionless diffusion length of the upstream and ionosphere plasmas is $l_{d, u P}=0.00125$, whereas for Io's body the diffusion length is $l_{\mathrm{d}, \mathrm{Io}}=0.0125$, where 
$l_{\mathrm{d}}=1 / R e$ and the magnetic Reynolds number is $R e=4 \pi U_{0} L \sigma_{\text {eff }} / c^{2}$. The average Lunquist number for the ionosphere may be estimated as

$$
L q=\frac{\tau_{d i f}}{\tau_{\mathrm{A}}}=\frac{R e}{M_{\mathrm{A}}}
$$

where the characteristic diffusion and Alfvén times are $\tau_{d i f}=4 \pi \sigma L^{2} / c^{2}$ and $\tau_{\mathrm{A}}=L / v_{\mathrm{A}}$. Note that Linker et al. (1998) used the following Lundquist number, $L q=500-1000$, magnetic Reynolds number, $R e=200-400$ and diffusion length, $l_{\mathrm{d}}=0.00125$ for the background plasma. This value of the diffusion length corresponds to an effective conductivity of about $1.2 \times 10^{8} \Omega^{-1} \mathrm{~m}^{-1}$ far in upstream. Since many plasma and atmosphere parameters are still uncertain we have to study a wide spectrum of simple models in order to choose the best one for interpretation of the observational data. We can present here only a sample of the wide spectrum simulation results which are in agreement or in disagreement with observations in order to illustrate the dependence of the plasma environment near Io on the input parameters of the problem. The global structure of Io's environment is determined by a set of dimensionless independent parameters such as $M_{\mathrm{A}}, \beta_{\mathrm{p}}, \beta_{\mathrm{e}}, M_{\mathrm{PI}} / M_{\mathrm{p}}$, ion production and charge exchange rates, diffusion lengths, and the ion gyroradius $\epsilon=\rho_{\mathrm{ci}} / L$. For real values of the magnetic field the value of the ion gyroradius is about $8 \mathrm{~km}$ which is calculated by the use of the local bulk velocity. The dimensionless ion gyroradius and grid spacing have the values $\epsilon=0.0045$ and $\Delta_{x} / L=0.1$. In order to study the ion kinetic effect we have to resolve the ion gyroradius on the grid. For this reason we use the artificially increased value of this parameter, $\epsilon=0.126$, that is about of a value of $\Delta_{x} / L$ now. Although, such way 
allows us to study the some kinetic effects for realistic physics we have to extrapolate our results into a realistic scale. By scaling the gyroradius in such a way, we preserve the ratio of $M_{\mathrm{A}}, M_{\mathrm{S}}, \beta_{\mathrm{p}}, \beta_{\mathrm{e}}$, and more importantly accurately preserve any anisotropy of the ion distribution function with respect to the magnetic field.

\subsection{Global Structure of the Io's Environment}

Let us consider first the global picture of the interaction of the plasma torus with Io in a case with an ionization rate $Q_{\text {ion }}=3.03 \times 10^{27} \mathrm{~s}^{-1}$ (run ceie2, Table 1) and charge exchange of form $\frac{A}{r^{2}}+B \exp \frac{r-r_{10}}{H_{\text {atmos }}}$. The maximum value of the atmosphere density is $n_{\text {atmos }}=5 \times 10^{7} \mathrm{~cm}^{-3}$ and diffusion lengths are $l_{\mathrm{d}, \mathrm{up}}=0.00125$ and $l_{\mathrm{d}, \mathrm{Io}}=0.00125$.

Figures 2 and 3 demonstrate the asymmetrical distribution of the torus plasma ion (top) and pickup ion density (bottom) in the $x-z$ and $y-z$ planes. One can see the increase of the incoming ion density upstream of Io. The pickup ion motion is determined mainly by the electromagnetic drift. The motion along the magnetic field is due to the thermal velocity and the gradient of the electron pressure. The asymmetrical distribution of the incoming ions in the $y-z$ plane may be explained by an existence of the $B_{z}$ component of the upstream magnetic field. The inclination of the magnetic field results in the asymmetrical boundary condition for ion dynamics (penetration and reflection) in the Io's ionosphere and the asymmetrical Alfvén wing. The incoming ions flow around the Alfvén wing so that only a small portion of the incoming flux penetrates the wing, resulting in a decrease in the incoming ion density. This effect is stronger in upper half-plane $(y>0)$ (Fig. 3, top) because the Alfvén wing has a stronger 
front transition in this region than in a lower half-plane. The pickup ion distribution,

Fig. 3 (bottom), gives the correct value for the inclination of the Alfvén wing, 21-21.5 degrees. The density profile is a little bit disturbed near the side boundaries, however, this perturbation does not affect the region close to Io as discussed already. Figures 4 and 5 show the distribution of the electric and magnetic fields. The asymmetry of the distributions in $\mathbf{E}$ and $\mathbf{B}$ appears to be caused by finite gyroradius effects of incoming and pickup ions. A weak perturbation of the magnetic field was observed near the ionosphere of Io: compression of the magnetic field upstream and decompression in the plasma wake.

Figure 5 also shows the formation of a strong Alfvén (and whistler) wing in the direction of the magnetic field. The perturbation of the electric field inside the wings is very strong and it may affect ion dynamics so that particles flow around the wings. The formation of the Alfvén wing in a subalfvénic flow near Io was studied first analytically by Neubauer (1980). A excitation of a whistler wave near a plasma cloud was studied by using 3-D hybrid simulation in Lipatov (2002). The above wave propagation is closely connected with the generation of low frequency waves by the harmonic dipole (local source) in the magnetized plasma. The first analytical studies of these effects may be found, for example in (Van'yan and Lipatov, 1972, and references therein).

Figures 6 and 7 show the velocity arrows of incoming and pickup ion velocities. The incoming ions flow around the effective obstacle that is produced by pickup ions and the ionosphere. The pickup ions flow from the "corona" across the magnetic field due to electromagnetic drift whereas the motion along the magnetic field is determined by the 
thermal velocity of ions and the electron pressure. Figure 8 shows the two-dimensional cross section for total ion density in the $x-y$ plane. One can see the asymmetry of distribution relative to the $x$-axis due to the angle between the bulk velocity and the magnetic field upstream and to the $y$-axis due to effects of the finite ion gyroradius.

\subsection{Effect of Electron Temperature on the Plasma Environment}

Our model takes into account the three characteristic electron temperatures that do not play any role in past MHD models: a) the temperature of electrons in the plasma torus, $T_{\mathrm{e}, \mathrm{up}}$; b) the temperature of electrons that are created together with pickup ions, $\left.T_{e, \mathrm{PI}} ; \mathrm{c}\right)$ the temperature of electrons that are created with ions in the ionosphere,


exosphere, i.e $W_{\text {ext }}=0$.

Let us consider the case (a). Figure 9 shows the one-dimensional cuts of the total ion density, temperature and magnetic field along the $x$-axis for $z=1.5 \times r_{\mathrm{Io}}$ and $y=0$. One can see two peaks in the distribution of the density, each of which has a width of about $r_{\text {Io }}$ and maximum densities of about 4 and 6.5 relative to the upstream density. The depletion of the density at $x=2 L$ may be explained by the following. When the supersonic flow passes around the obstacle, a wake with a decreased density is formed. The simple example of a such void is presented by the lunar plasma wake. However, in our case an asymmetrical pickup ion high density obstacle provides an asymmetrical void in Io's plasma wake. The temperature profile has two peaks with maximum temperatures of about 2.3 and 0.7 . These peaks do not correlate with the 
peak in density because the temperature of the ions is determined by the heated ions from the incoming flow and pickup ions. The magnetic field profile shows a decrease in the magnetic field with a minimum value of about 0.5 in the plasma wake and with some significant oscillations (Fig. 9). Figure 10 shows a two-dimensional cross section of the total ion density across the plasma wake $\left(z=1.5 \times r_{\mathrm{lo}}\right)$. One can see three strong maxima. This distribution is determined by finite gyroradius effects of ions.

If we take into account the temperature of electrons that are created with pickup ions (case (b)) the distribution of the ions in the plasma wake may be changed significantly. The one-dimensional total density profile has one peak with a value of about 8 (Fig. 11). The temperature profile has also one strong peak with a value of about 1.9 and the magnetic field has a depletion with a minimum value 0.7 (Fig. 11). Figure 12 shows a two-dimensional cross section of the total density across the plasma wake. One can see a strong peak narrow in $x$-direction and wide in $y$-direction.

In case (c), when the temperature of electrons inside the ionosphere is also taken into account, we have the following distribution of plasma parameters in the wake. Figure 13 shows one-dimensional cuts of the total density, temperature and magnetic field. The density profile has a wide peak with a thickness of about $4 r_{\text {Io }}$ and a peak value of $n \approx 6$. The temperature profile has a two-peak distribution. The value of the temperature at the two peaks is about 0.6 and 0.55 . The peaks are separated by a distance of $4 \times r_{\mathrm{Io}}$. The magnetic field profile has a minimum at the plasma wake with $B=0.55$ (Fig. 13). Figure 14 shows the two-dimensional cross section of the total density across the plasma wake. One can see a strong peak which is narrow in 
the $y$-direction and wide in the $x$-direction. By introducing cool electrons inside the ionosphere we are trying to account in an appropriate way for the cooling effect on electrons through electron-collisions close to Io.

Finally, we show the two-dimensional cuts of pickup ion density for all three cases, Fig. 15 (top, middle, bottom). One can see that the temperature of electrons which are created in different regions of Io's environment may affect strongly the pickup ion distribution. Figure 15 demonstrates the importance of the electron pressure in the region close to the Io.

In the case of the absence of pressure of pickup and ionospheric electrons, the interaction of the plasma torus ions with Io is strongly asymmetrical in the $x$ - $z$ plane due to the finite ion gyroradius. In the plasma wake a "space-mixing" of the pickup ions is observed that results in the formation of an asymmetrical tongue-type distribution in the $x-z$ plane. The effusion of pickup ions in the $y$ direction is also weak because of small values of $\Delta p_{e}$ and the velocity of the pickup ions in $y$ direction, Fig. 15, (top).

In the opposite case, when the gradient of the pressure of the pickup electrons and the pressure of ionospheric electrons cause a strong electrostatic field, the pickup ion density distribution in the $x-z$ plane becomes more symmetrical and wider across the wake compared with the above case (cf. Fig. 15 (top) and (bottom)). The gradient of the electron pressure also causes a strong effusion of the pickup ions along the Alfvén wing (see, Fig. 15 (bottom) and (top)).

Finally, in the intermediate case when the pressure of ionospheric electrons is small the asymmetrical distribution of the pickup ion density with a strong effusion along the 
Alfvén wing is observed, Fig. 15 (middle). So, we can conclude that the pressure of the pickup ions is responsible for effusion processes while the pressure of the ionospheric electrons is responsible for the formation of the symmetrical distribution with the maximum in density located near the $(y=0)$ plane.

All three cases, (a), (b) and (c), demonstrate strong asymmetries in the distributions of pickup ion density, temperature and magnetic field in comparison with MHD and electrodynamic models.

\subsection{Effect of Pickup Ion Injection Distribution on the Plasma Environment}

To study the role of pickup ions from the halo we simulated Io's environment for cases when the ion production rate in the halo corresponds to $W_{\text {ext }}=10 \%$ and $W_{\text {ext }}=30 \%$ of the total (halo plus exosphere) ion production rate. Note that all other parameters are the same in these cases.

Figure 16 (solid line) presents one-dimensional cuts for density, temperature and magnetic field for $W_{\text {ext }}=10 \%$ and $\beta_{\mathrm{e}, \text { iono }}=0.25 \beta_{\mathrm{e}}$. The density profile has a maximum value of about $n \approx 7.2$ with a characteristic width of the peak of $3 \times r_{\mathrm{Io}}$, that is higher than the observational value, $n \approx 5.1$. The temperature profile shows a two-peak distribution. The maximum values in the peaks are $T / T_{0} \approx 3$ and $T / T_{0} \approx 2.1$ that correspond to the observational data (Frank et al., 1996; Kivelson et al., 1996). The distance between peaks is about $3 \times r_{\mathrm{Io}}$ that is a little bit wider than observed value, $2 \times r_{\mathrm{Io}}$. The magnetic field profile has a minimum value in the plasma wake of $B \approx 0.5$ that also corresponds the minimum value of the $B_{y}$ in the observational data. Figure 16, 
(dotted line) shows the one-dimensional cuts for density, temperature and magnetic field for $W_{e x t}=30 \%$ and $\beta_{e, \text { iono }}=0.25 \beta_{e}$. The density profile has a maximum value of about $n \approx 5.83$ with a characteristic width of the peak of $4 \times r_{\text {Io }}$, which is a little bit wider than that in Fig. 16, (solid line) and is a little higher than observed. The temperature profile also shows a two-peak distribution. The maximum values in the peaks are $T / T_{0} \approx 6.8$ and $T / T_{0} \approx 2.4$. Note that the first maximum in the temperature is higher than observed (Frank et al., 1996; Kivelson et al., 1996). The magnetic field profile shows a minimum value of the magnetic field in the plasma wake $B \approx 0.56$ that corresponds the observational data.

\subsection{Effect of Ionospheric Conductivity on the Plasma Environment}

In the previous cases we have assumed a high conductivity for the ionosphere and Io's body. The realistic models of Io may include a conducting core, surrounded by a poorly conducting mantle (a Moon-like model), or Io may be considered as a poorly conducting body. In this section we shall model the Io as a poorly conducting body. Note that all other parameters in this case are the same as that shown in Fig. 16, (solid line) except the higher diffusion length. Figure 16 (dashed line) shows one-dimensional cuts for the density, temperature and magnetic field for the case with a diffusion length, $l_{d, \text { iono }}=0.025$. Note that the lines in Fig. 16 corresponds the simulation for different sizes of the computational domain. The value of the peak density is about $n \approx 7.5$ with the same thickness of the peak, $\approx 3 r_{\text {Io }}$ as in Fig. 16 , (solid line). The maximum values of the peak temperature, 3 and 2 are approximately the same as 
in Fig. 16, (solid line) (see Fig. 16, (dashed line)). However, the magnetic field profile shows much stronger field variation in the external region of the plasma wake than in Fig. 16, (solid line). The analysis of the magnetic field inside Io shows that the reduced conductivity $\left(l_{\mathrm{d} \text {,iono }}=0.025\right.$, Fig.18 (dashed line) $)$ near ionosphere and inside Io may reduce the magnetic field inside Io by $20-40 \%$ and the peak plasma density at the Galileo trajectory by $5 \%$ in comparison with the model with $\left.l_{\mathrm{d}, \text { iono }}=0.0025\right)$.

\subsection{Comparison with Galileo Observational Data}

The result of the measurements by the particle and field instruments on the Galileo Orbiter during the December 1995 flyby of Io provided new and important information with which realistic simulations for the plasma interaction can be tested. Along that trajectory physical signatures of the wake were seen as a broad depression in the magnetic field (Kivelson et al., 1996), sharp peaks in the ion (Frank et al., 1996) and electron (Gurnett et al., 1996) densities, a slowing of the plasma in the core of the wake, a deep ion temperature decrease in the center of the wake, and a large (factor of 3 ) ion temperature rise in the flanks of the wake (Frank et al., 1996). The magnetic field perturbation was broader spatially than the density peak, and showed a double-reversed structure, whereby the perturbation (as defined by the difference from the outer Jovian B-field value) was actually weaker right near the close-approach point than it was somewhat adjacent to the center of the wake. For comparison of the our computational model with observation data we made a run with the following plasma parameters: $\beta_{\mathrm{e}, \mathrm{PI}}=0.125 \beta_{\mathrm{e}, \mathrm{up}}, \beta_{\mathrm{e}, \text { iono }}=\beta_{\mathrm{e}, \mathrm{up}}, n_{\mathrm{iono}}=100 n_{0}, H_{\mathrm{atmos}}=0.06 r_{\mathrm{Io}}, W_{\text {ext }}=5 \%$, and 
$l_{\mathrm{d}, \mathrm{up}}=0.00125$. The conductivity of Io is chosen so that $l_{\mathrm{d}, \mathrm{Io}}=0.0125$.

Let us consider first a case in the absence of charge exchange processes (case nce Table 1). The total ion production rate was $Q_{\text {ion }}=4.8 \times 10^{27} \mathrm{~s}^{-1}$. Fig. 17 shows the comparison of plasma parameters and magnetic field components from the simulation model and observation along the Galileo trajectory (Pass I0). Note that we used the interpolation of the grid values of the plasma parameters and the magnetic field into the position of Galileo spacecraft. We can see that the density peak is slightly shifted to the right in comparison with observed one. The left peak in the temperature profile is a little bit narrower than the observed one while the right peak is a little bit higher than the observed one. The simulation yields a little bit smaller value of $B_{y}$ at large $x, x>>r_{\text {Io }}$, due to perturbation of the electromagnetic field in the region above $(y>0)$ and below $(y<0)$ the equatorial plane. Unlike the MHD models the hybrid model yields a $B_{y}$ profile with reverse structure in the middle of the plasma wake. This type of behavior may be explained by the diamagnetic and accelerational drift currents in the plasma wake, which are modeled naturally in our kinetic description for ions. The maximum value of these currents is located near the equatorial plane in the boundary layer (interface) between the external plasma flow and pickup ions in the plasma wake. The $B_{z}$ profiles in these simulations correspond very well to the observed data however the left maximum in the magnetic field is smaller than observed one. Unfortunately fluctuations in magnetic field components are not very small due to "shot" noise. So the lack in agreement between observed and computed values of $B_{x}$ is probably just the result from less than perfect plasma simulation model ("shot" noise), 
and the simplified models of the ionosphere and Io's body.

Let us consider now the cases with a charge exchange rate that corresponds to the maximum value of the density of the atmosphere, $n_{\text {atmos }}=10^{8} \mathrm{~cm}^{-3}$. We assume here that the charge exchange process is due to only the exponential part of the neutral atmosphere.

Fig. 18 (solid line) shows the comparison of plasma parameters and magnetic field components from our simulation model and the observations in the case with a total ion production rate, $Q_{\text {ion }}=2.27 \times 10^{27} \mathrm{~s}^{-1}$. In this case the density profile has a smaller effective value but the total temperature is a little bit higher. The left peak in the temperature profile is also a little bit narrower than in the observational data while the right peak is a little bit higher than the observed one. The perturbation in $B_{y}$ is a little bit smaller than in the case of absence of charge exchange, (cf. Fig. 18 (solid line) and Fig. 17).

In case of higher total ion production rate, $Q_{\text {ion }}=2.57 \times 10^{27} \mathrm{~s}^{-1}$ (Fig. 18, (dotted line)) the density profile has a higher effective value but the total temperature is a little bit lower. We can see that the density profile has a depletion on the left side and the width of the peak is smaller than in the case with no charge exchange, (cf. Fig. 18, (dotted line) and Fig. 17). The left peak in the temperature profile is also a little bit narrower than in the observational data while the right peak is a little bit higher than the observed one. The perturbation in $B_{y}$ is a little bit smaller than in case of absence of charge exchange (cf. Fig. 18, (dotted line) and Fig. 17).

In a case with a higher charge exchange rate that corresponds to the maximum 
value of the density of atmosphere, $n_{\mathrm{atmos}}=5 \times 10^{8} \mathrm{~cm}^{-3}$ and a total ion production rate, $Q_{\text {ion }}=2.1 \times 10^{27} \mathrm{~s}^{-1}$ we have a satisfactory agreement in the total density profile, a smaller total temperature and no agreement in the magnetic field component $B_{y}$.

Let us consider the cases with a high charge exchange rate that corresponds to the maximum value of the density of atmosphere, $n_{\text {atmos }}=10^{9} \mathrm{~cm}^{-3}$ and an ionization rate of $Q_{\text {ion }}=(1.4-2.1) \times 10^{27} \mathrm{~s}^{-1}$. In case of low ionization rate, $Q_{\text {ion }}=1.4 \times 10^{27} \mathrm{~s}^{-1}$, the density peak is higher and thinner than the observed one whereas the temperature profile is in a good agreement with the observation. The magnetic field $B_{y}$ profile is only in qualitative agreement with observation. In the case of a higher ionization rate, $Q_{\text {ion }}=2.1 \times 10^{27} \mathrm{~s}^{-1}$, the density has a strong double peak structure but the temperature profile is in agreement with the observations. However, the magnetic field profile has no agreement with observational data.

In previous cases we assumed that the charge exchange process is only due to the lower altitude, exponential part of the neutral atmosphere. Let us consider now the results of simulation that includes also the charge exchange due to the extended atmosphere which is distributed as $r^{-2}$. Figure 19 shows the profiles of the total density, temperature and magnetic field for different maximum values of the density of atmosphere, $n_{\text {atmos }}=\left(5 \times 10^{7}-10^{9}\right) \mathrm{cm}^{-3}$, and an ionization rate, $Q_{\text {ion }}=(3.03-4.04) \times 10^{27} \mathrm{~s}^{-1}$.

Let us consider first the cases with a low maximum value of the density of atmosphere, $n_{\text {atmos }}=5 \times 10^{7} \mathrm{~cm}^{-3}$, and an ionization rate of $Q_{\text {ion }}=(3.03-4.04) \times 10^{27} \mathrm{~s}^{-1}$. In case of lower ionization rate, $Q_{\text {ion }}=3.03 \times 10^{27} \mathrm{~s}^{-3}$, the density profile is in agreement 
with the observation, but the temperature is a little bit higher in the peaks (Fig. 19, solid line). The magnetic field profile $\left(B_{y}\right)$ is in a qualitative agreement with the observation (Fig. 19, solid line). In the case of higher ionization rate, $Q_{\text {ion }}=4.04 \times 10^{27} \mathrm{~s}^{-1}$, the density profile is a little bit higher in peak than the observed one and the temperature is a little bit lower in the left peak. The magnetic field profile $\left(B_{y}\right)$ is much smoother than in the observation (not shown in Fig. 19).

In the cases with a higher maximum atmosphere density, $n_{\text {atmos }}=10^{8} \mathrm{~cm}^{-3}$, the maximum value of the wake density becomes much higher than in the observation. Although the temperature profiles are in good agreement with the observations, the magnetic field component $B_{y}$ is much smoother in the case of $Q_{\text {ion }}=3.03 \times 10^{27} \mathrm{~s}^{-1}$ (Fig. 19, (dotted line)), and it is in qualitative agreement with the observations in the case with $Q_{\text {ion }}=4.04 \times 10^{27} \mathrm{~s}^{-1}$ (not shown in Fig. 19). The increase in the atmosphere maximum to $n_{\text {atmos }}=5 \times 10^{8} \mathrm{~s}^{-3}$ results in an increase of the density to more than twice the observed value. In this case we have $Q_{\text {ion }}=4.04 \times 10^{27} \mathrm{~s}^{-1}$ with a total charge exchange rate of $Q_{\text {exch }}=5.8 \times 10^{28} \mathrm{~s}^{-1}$, Fig. 19, (dashed line).

In case of a high maximum atmosphere density, $n_{\text {atmos }}=10^{9} \mathrm{~cm}^{-3}$ there is no similarity between simulation and observation. We simulated the cases with ionization rates, $Q_{\text {ion }}=(2.1-4.04) \times 10^{27} \mathrm{~s}^{-1}$. In the case of low ionization rate, $Q_{\text {ion }}=2.1 \times 10^{27} \mathrm{~s}^{-1}$, the density profile has two thin peaks (Fig. 19, (dot-dashed line)) while the magnetic field profile $\left(B_{y}\right)$ has a strong variation (Fig. 19, (dot-dashed line)) that does not correspond the observation. In case of moderate ionization rate, $Q_{\text {ion }}=3.03 \times 10^{27} \mathrm{~s}^{-1}$, the density profile has one peak with a value, $n_{\text {total }}>10 n_{0}$ 
that is much higher than observed one whereas the magnetic field profile $\left(B_{y}\right)$ is much smoother than observed one. In the case of a high ionization rate, $Q_{\text {ion }}=4.2 \times 10^{27} \mathrm{~s}^{-1}$, the density profile has one peak with a value, $n_{\text {total }} \approx(8-10) n_{0}$ that is much higher than observed whereas the magnetic field profile $\left(B_{y}\right)$ is much smoother than observed.

One of the main issues of the plasma torus - Io interaction is the question about the real obstacle in this interaction. There are two possible candidates for this problem - (1) mass loading by the pickup ions originally produced from Io's atmosphere by the ionization processes or charge exchange processes between the incoming plasma torus ions and the neutral atmosphere, or (2) charge exchange processes between the pickup ions originally produced from Io's atmosphere and the neutral atmosphere. Table 1 gives a summary of results of simulations for different models that are considered in this section.

In the case of the models with a high density of Io's atmosphere $\left(10^{9} \mathrm{~cm}^{-3}\right)$ the charge exchange rate becomes very high, $Q_{\text {exch }} \approx(6.7-9) \times 10^{28} \mathrm{~s}^{-1}$ (cases ceie6, ceie7 and seie8, Table 1). Note that in these cases the charge exchange rate for incoming ions is much smaller than for pickup ions. We compared the charge exchange rate in the external $\left(r>4 H_{\text {atmos }}\right)$ and the internal $\left(r \leq 4 \times H_{\text {atmos }}\right)$ region. The charge exchange rate for incoming ions in the external region has approximately the same value as a charge exchange rate in the internal region. However, the charge exchange rate for pickup ions in the external region is approximately 20 times smaller than the charge exchange rate in the internal region.

The magnetic field profile $\left(B_{y}\right)$ does not match the observational data very well 
for a small ionization rate, $Q_{\text {ion }}=2.1 \times 10^{27} \mathrm{~s}^{-1}$ (case ceie3, Table 1), whereas the total density value in the plasma wake is much more than observed one in the case of higher ionization rate, $Q_{\text {ion }}=(3.03-4.04) \times 10^{27} \mathrm{~s}^{-1}$ (cases ceie1 and ceie2, Table 1). Hence, the models with a high charge exchange rate, $Q_{\text {exch }}=(6.5-9) \times 10^{28} \mathrm{~s}^{-1}$, are not realistic because they cannot explain the observational data.

The model with $n_{\text {atmos }}=5 \times 10^{8} \mathrm{~cm}^{-3}$ (case ceie5, Table 1), gives the total charge exchange rate $Q_{\text {exch }}=5.8 \times 10^{28} \mathrm{~s}^{-1}$. The models with $n_{\text {atmos }}=10^{8} \mathrm{~cm}^{-3}$ (cases ceie3 and ceie4, Table 1), give total charge exchange rates of $Q_{\text {exch }}=(1.09-1.35) \times 10^{28} \mathrm{~s}^{-1}$ And, finally, the models with $n_{\text {atmos }}=5 \times 10^{7} \mathrm{~cm}^{-3}$, give total charge exchange rates of $Q_{\text {exch }}=(6.12-6.54) \times 10^{27} \mathrm{~s}^{-1}$ (cases ceie1 and ceie2, Table 1).

The analysis of the models shows that two cases that have a good fit for observational data. In the model without charge exchange (case nce, Table 1) the simulation results describe well enough the main observational data - a total density, a total temperature and the magnetic field $\left(B_{y}\right)$ (Fig. 17). The more realistic model with $n_{\text {atmos }}=5 \times 10^{7} \mathrm{~cm}^{-3}$, and ionization rate $Q_{\text {ion }}=3.03 \times 10^{27} \mathrm{~s}^{-1}$ (case ceie1, Table 1), also fits satisfactorily the observational data (Fig. 19, solid line). This model gives the charge exchange rates $Q_{\mathrm{p}, \text { exch }}=3.16 \times 10^{27} \mathrm{~s}^{-1}$ and $Q_{\mathrm{c}, \text { exch }}=3.13 \times 10^{27} \mathrm{~s}^{-1}$ for plasma torus ions and pickup ions respectively and would correspond to an MHD model with total fresh ion mass-loading rate and the charge exchange rate, which contributes to the momentum and energy friction terms, of $1.2 \times 10^{28} \mathrm{~s}^{-1}$ (Combi, Gombosi and Kabin, 2002). So, we find that the interaction is dominated in roughly equal parts between primary torus ion charge exchange and secondary pickup ion charge exchange. 


\section{Conclusions}

3D Boltzmann simulations of the interaction of the Jovian plasma torus with Io, have demonstrated several new features:

- The whistler (lead front) and quasi-stationary Alfvén waves (wings) strongly affect plasma flow around Io.

- The effect of the finite ion gyroradius that results in formation of the asymmetrical boundary layer in the vicinity of Io's ionosphere is important. The plasma parameters have a strongly asymmetrical distribution across Io's wake. The kinetic behavior of ion dynamics reproduces the inverse structure of the magnetic field (due to drift current) which cannot be explained by standard MHD or electrodynamic simulations which do not account for anisotropic ion pressure. The diamagnetic effect of non-isotropic gyrating pickup ions broadens the B-field perturbation and produces increased temperatures in the flanks of the wake, as observed by the Galileo spacecraft, but not explained by previous simulations. Note that two-fluid simulation (Saur et al., 2002) produces the double-peak signature with spatial scale much smaller than in observation.

- The cold, dense wake is produced, as in MHD, but was not produced in electromagnetic simulations without ad hoc addition of bidirectional electrons.

- The values of temperatures of the electrons which are created and cooled by 
collision with neutrals in the exosphere and inside the ionosphere may strongly affect the pickup ion dynamics along the magnetic field and consequently the pick up distribution across the wake. In the absence of an observed global picture of the plasma distribution, the simulation serves to demonstrate the wide range of global configurations that are possible for various electron temperature descriptions. A full hybrid simulation for the plasma including an electron temperature treatment that accounts for electron-neutral collision and a detailed neutral description would be required to produce an accurate global picture from the first principles.

- The effective conductivity of Io's ionosphere may change strongly the distribution of the magnetic field near and inside Io. The reduced conductivity $\left(l_{d i f}=0.025\right)$ near the ionosphere and inside the Io may reduce the magnetic field inside Io by $20-40 \%$ and the peak of the plasma density at the Galileo trajectory by $15 \%$.

- The best models that fit the observational data well are: the model without charge exchange and ionization rate $Q_{\text {ion }}=4.8 \times 10^{27} \mathrm{~s}^{-1}$, and the model with ionization rate $Q_{\text {ion }}=3.03 \times 10^{27} \mathrm{~s}^{-1}$ and the total charge exchange rate $Q_{\text {exch }}=6.3 \times 10^{27} \mathrm{~s}^{-1}$. The best MHD model had a total fresh ion mass-loading rate of $6 \times 10^{27} \mathrm{~s}^{-1}$ and a charge exchange rate, which contributes to the momentum and energy friction terms, of $1.2 \times 10^{28} \mathrm{~s}^{-1}$ (Combi, Gombosi and Kabin, 2002). The reason for the factor of 2 difference results from the above-described pressure anisotropy effect in the kinetic simulation, and the resulting tighter plasma distribution near the equator plane defined by Io. 
- In the presented calculations the value of the ion gyroradius was much larger than the realistic value. So we have to investigate the effects of realistic values of the ion gyroradius in future simulations. We expect that the smaller ion gyroradius may result in smaller asymmetry of the global picture. However, we do not expect the strong changes in the plasma and the magnetic field profiles because the used value of the ion gyroradius is much smaller than the characteristic scale of the problem - the radius of Io. These profiles are controlled primary by the inter-penetration of the torus plasma and the pickup ions.

Acknowledgments. A.S.L. and M.R.C. were supported in part by the NASA Grant NAG5-9464 from the Applied Information Systems Research Program and NAG5-12822 from The Planetary Atmospheres Program. Computational resources were provided by the San Diego Super Computer Center (IBM Blue-Horizon), the Center for Advanced Computing Univ. of Michigan (IBM POWER 3 Cluster), the National Partnership for Advanced Computational Infrastructure, and the Computer Center TU of Braunschweig (Alpha-Cluster). The authors thank Andreas Kopp and other referee for their helpful comments. A.S.L thanks D. Markiewicz-Innes for her helpful comments. 


\section{References}

Braginskii, S.L., Transport processes in a plasma. In: Leontovich, M.A. (Ed.), Reviews of Plasma Physics. Consultants Bureau, New York, pp. 205-240.

Cloutier, P.A., Daniell, R.E., Dessler, A.J., and Hill, T.W., 1978. A cometary ionosphere model for Io. Astrophys. Space Sci. 55, 93-112.

Combi, M.R., Gombosi, T.I. and Kabin, K., 2002. Plasma Flow Past Cometary and Planetary Satellite Atmospheres. In: Mendillo, M., Nagy, A., Waite, J.H. (Eds.), Atmospheres in the Solar System: Comparative Aeronomy. Geophys. Monograph Series Vol. 130. AGU Washington, D.C., pp. 151-167.

Combi, M.R., Kabin, K., Gombosi, T., De Zeeuw, D.L., and Powell, K., 1998. Io's plasma environment during the Galileo flyby: Global three-dimensional MHD modeling with adaptive mesh refinement. J. Geophys. Res. 103, 9071-9081.

Frank, L.A., Paterson, W.R., Ackerson, K.L., Vasyliunas, V.M., Coroniti, F.V., and Bolton, S.J., 1996. Plasma observations at Io with the Galileo spacecraft. Science, 274, 394-395.

Frank, L.A., and Paterson, W.R., 2000. Return to Io by the Galileo spacecraft: Plasma observation. J. Geophys. Res. 105, 25363-25378.

Gurnett, D.A., Kurth, W.S., Roux, A., Bolton, S.J., Kennel, C.F., 1996. Galileo Plasma Wave Observations in the Io Plasma Torus and Near Io. Science. 274, 391-392. 
Goertz, C.K., 1980. Io's interaction with the plasma torus. J. Geophys. Res. 85, 2949-2956.

Ip, W.-H., 1990. Neutral gas-plasma interaction: The case of the Io plasma torus. Adv. Space Res. 10(1), 15-18.

Kabin, K., Combi, M.R., Gombosi, T.I., DeZeeuw, D.L., Hansen, K.S., and Powell, K.G., 2001. Io's magnetospheric interaction: an MHD model with day-night asymmetry. Planetary and Space Sci. 49, 337-344.

Kivelson, M.G., Khurana, K.K., Walker, R.J., Warnecke, J., Russell, C.T., Linker, J.A., Southwood, D.J., and Polanskey, C., 1996. Io's interaction with the plasma torus: Galileo magnetometer report. Science 274, 396-398.

Kivelson, M.G., Khurana, K.K., Russell, C.T., Joy, S.P., Volwerk, M., Walker, R.J., Zimmer, Ch., and Linker, J.A., 2001. Magnetized or unmagnetized: Ambiguity persists following Galileo's encounters with Io in 1999 and 2000. J. Geophys. Res. $106,26121-26136$.

Kopp, A., 1996. Modifications of the electrodynamic interaction between Jupiter and Io due to mass loading effects. J. Geophys. Res. 101, 24943-24954.

Kopp, A., Birk, G.T., Otto, A., 1998. On the formation of Io-induced acceleration region related to Jovian aurora. Planet. Space Sci. 46, 405-415.

Lellouch, E., 1996. Urey Prize Lecture. Io's Atmosphere: not yet understood. Icarus $124,1-21$. 
Linker, J.A., Khurana, K.K., Kivelson, M.G., and Walker, R.J., 1991. A threedimensional MHD simulation of plasma flow past Io. J. Geophys. Res. 96, 21037-21053.

Linker, J.A., Khurana, K.K., Kivelson, M.G., and Walker, R.J., 1998. MHD simulation of Io's interaction with the plasma torus. J. Geophys. Res. 103(E9), 19867-19877.

Linker, J.A., Kivelson, M.G., and Walker, R.J., 1989. The effect of mass loading on the temperature of a flowing plasma. Geophys. Res. Lett. 16(7), 763-766.

Lipatov, A. S., 2002. The Hybrid Multiscale Simulation Technology. An introduction with application to astrophysical and laboratory plasmas. Springer-Verlag, Berlin, Heidelberg and New York.

Lipatov A.S., Zank, G.P., Pauls, H.L., 1998. The Dynamics of an $H$ Neutral Component inside the Heliosphere: 2.5D Particle-Mesh Boltzmann Simulation. J. Geophys. Res. 103, 20636, 1998.

Malama, Y.G., 1991. Monte-Carlo simulation of neutral atoms trajectories in the solar system. Astrophys. Space Sci. 176, 21-39.

Neubauer, F.M., 1980. Nonlinear standing Alfvén wave current system at Io - Theory. J. Geophys. Res. 85, 1171-1178.

Ripkin, H.L., Fahr, H.J., 1983. Modification of the local interstellar gas properties in the heliospheric interface. Astron. astrophys. 122, 181-190.

Saur, J., Neubauer, F.M., Strobel, D.F., and Summers, M.E., 1999. Three-dimensional 
plasma simulation of Io's interaction with the Io plasma torus: Asymmetric plasma flow. J. Geophys. Res. 104, 25105.

Saur, J., Neubauer, F.M., Strobel, D.F., and Summers, M.E., 2002. Interpretation of Galileo's Io plasma and field observations: I0, I24, and I27 flybys and close polar passes. J. Geophys. Res. 107, 1422.

Saur, J., Strobel, D.F., Neubauer, F.M., and Summers, M.E., 2003. The ion mass loading rate at Io. Icarus $163(2), 456-468$.

Southwood, D.J., and Dunlop, M.W., 1984. Mass pickup in sub-Alfvénic plasma flow: A case study for Io. Planet. Space Sci. 32, 1079-1089.

Southwood, D.J., Kivelson, M.G., Walker, R.J., and Slavin, J.A., 1980. Io and its plasma environment. J. Geophys. Res. 85, 5959-5968.

Umeda, T., Omura, Y., Matsumoto, H., 2001. An improved masking method for absorbing boundaries in electromagnetic particle simulations, Comp. Phys. Comm. (137), 286-299.

Van'yan, L.L. and Lipatov, A.S., 1972. Three-dimensional hydromagnetic disturbances generated by a magnetic dipole in an anisotropic plasma. Geomagn. and Aeronomy 18(5), 316-318.

Williams, D.J., et al., 1996. Electron beams and ion composition measured at Io and its torus. Science. $274,401-403$. 
Williams, D.J., Thorne, R.M., and Mauk, B., 1999. Energetic electron beams and trapped electrons at Io. J. Geophys. Res. 104, 14739-14753.

Wolf-Gladrow, D.A., Neubauer, F.M., and Lussem, M., 1987. Io's interaction with the plasma torus: A self-consistent model. J. Geophys. Res. 92, 9949-9961.

Zueva, N.M., Imshennik, V.S., Lokutsievskii, O.B., Mikhailov, M.S., 1975. The model of nonhydrodynamical stage of plasma focus. Preprint No. 73. Keldysh Institute of Applied Mathematics, Moscow.

A. S. Lipatov and M. R. Combi, Space Physics Research Laboratory, Department of Atmospheric, Oceanic and Space Sciences, The University of Michigan, Ann Arbor, MI 48109-2143, USA, (emails: alipatov@engin.umich.edu, mcombi@umich.edu)

Received

To appear in the ICARUS, 2004 


\section{Figure captions}

Figure 1. Galileo trajectory close to Io and the system of coordinates.

Figure 2. Incoming (top) and pickup (bottom) ion density in the $x$ - $z$ plane. The case with $Q_{\text {ion }}=3.03 \times 10^{27} \mathrm{~s}^{-1}, n_{\text {atmos }}=5 \times 10^{7} \mathrm{~cm}^{-3}, \beta_{e, P I}=\beta_{e}, \beta_{e, \text { iono }}=0.25 \beta_{e}$ $W_{e x t}=5 \%$, and $H_{\text {atmos }}=0.06$. See explanation in Fig. 6.

Figure 3. Incoming (top) and pickup (bottom) ion density in the $y-z$ plane for the same parameters as Fig. 2. See explanation in Fig. 7.

Figure 4. Electric (top) and magnetic (bottom) fields in the $x-z$ plane for the same parameters as Fig. 2. Figure shows a strong asymmetry of the electromagnetic field due to finite gyroradius effects.

Figure 5. Electric (top) and magnetic (bottom) fields in the $y$ - $z$ plane for the same parameters as Fig. 2. Figure shows the formation of an Alfvén wing in the direction of the main magnetic field.

Figure 6. Incoming (top) and pickup (bottom) ion velocity arrows in the $x$ - $z$ plane for the same parameters as Fig. 2. The figure demonstrates a flow of pickup ions from the "corona" across the magnetic field. The incoming ions flow around the effective obstacle that is produced by pickup ions and ionosphere.

Figure 7. Incoming (top) and pickup (bottom) ion velocity arrows in the $y$ - $z$ plane for the same parameters as Fig. 2. The figure demonstrates a strong expansion of pickup ion "corona" along the magnetic field line. The incoming ions flow around the region of extended "corona". 
Figure 8. Two-dimensional section for total density in the $x-y$ plane for the same parameters as Fig. 2.

Figure 9. Total density, temperature and magnetic field along the $x$-axis. The case with $\beta_{e, P I}=0, \beta_{e, i o n o}=0, W_{e x t}=0 \%$, and $H_{\text {atmos }}=0.06$.

Figure 10. Two-dimensional section for total density in the $x-y$ plane. The case with $\beta_{e, P I}=0, \beta_{e, \text { iono }}=0, W_{\text {ext }}=0 \%$, and $H_{a t m o s}=0.06$

Figure 11. Total density, temperature and magnetic field along the $x$-axis. The case with $\beta_{e, P I}=\beta_{e}, \beta_{e, \text { iono }}=0, W_{\text {ext }}=0 \%$, and $H_{a t m o s}=0.06$.

Figure 12. Two-dimensional section for total density in the $x-y$ plane. The case with $\beta_{e, P I}=\beta_{e}, \beta_{e, i o n o}=0, W_{e x t}=0 \%$, and $H_{a t m o s}=0.06$.

Figure 13. Total density, temperature and magnetic field along the $x$-axis. The case with $\beta_{e, P I}=\beta_{e}, \beta_{e, \text { iono }}=\beta_{e}, W_{e x t}=0 \%, H_{\text {atmos }}=0.06$

Figure 14. Two-dimensional section for total density in the $x-y$ plane. The case with $\beta_{e, P I}=\beta_{e}, \beta_{e, \text { iono }}=\beta_{e}, W_{e x t}=0 \%, H_{\text {atmos }}=0.06$

Figure 15. Two-dimensional sections for pickup ion density in the $x-y$ plane. The cases with (top) $\beta_{e, P I}=0, \beta_{e, \text { iono }}=0 ;$ (middle) $\beta_{e, P I}=\beta_{e}, \beta_{e, \text { iono }}=0 ;$ (bottom) $\beta_{e, P I}=\beta_{e}, \beta_{e, \text { iono }}=0.25 \beta_{e} . W_{e x t}=0 \%$, and $H_{a t m o s}=0.06$. Figure demonstrates asymmetry of pickup ion density across the wake.

Figure 16. Total density, temperature and magnetic field along the $x$-axis for $\beta_{e, P I}=\beta_{e}, \beta_{e, \text { iono }}=0.25 \beta_{e}, H_{\text {atmos }}=0.06, l_{d, u p}=0.0025 .\left(\right.$ solid line) $W_{e x t}=10 \%$, $l_{d, \text { iono }}=0.0025 ;\left(\right.$ dotted line) $W_{\text {ext }}=30 \%, l_{d, \text { iono }}=0.0025 ;($ dashed line $) W_{\text {ext }}=10 \%$, $l_{d, \text { iono }}=0.025$. 
Figure 17. Comparison of Galileo PLS (I0 pass) [Frank et al., 1996] and MAG [Kivelson et al., 1996] (the open circles) data with the Io hybrid model results in absence of charge exchange. $Q=4.8 \times 10^{27} \mathrm{~s}^{-1}, \beta_{e, P I}=0.125 \beta_{e}, \beta_{e, \text { iono }}=0.125 \beta_{e}, W_{\text {ext }}=5 \%$, $H_{\text {atmos }}=0.06$, and $n_{\text {iono }}=800(100) n_{0} \cdot l_{d, u p}=0.00125$ and $l_{d, I o}=0.0125$.

Figure 18. Same as Fig. 17, but with charge exchange $\left(B \exp \frac{r-r_{\perp 0}}{H_{a t m o s}}\right)$, $n_{\text {atmos }}=10^{8} \mathrm{~cm}^{-3}:$ (solid line) $Q=2.27 \times 10^{27} \mathrm{~s}^{-1} ;$ (dashed line) $Q=2.57 \times 10^{27} \mathrm{~s}^{-1}$.

Figure 19. Same as Fig. 17, but with charge exchange $\left(\frac{A}{r^{2}}+B \exp \frac{r-r_{/ 0}}{H_{a t m o s}}\right)$ : (solid line) $n_{\text {atmos }}=5 \times 10^{7} \mathrm{~cm}^{-3}, n_{\text {iono }}=n_{0}, Q=3.03 \times 10^{27} \mathrm{~s}^{-1}, Q_{p, \text { exch }}=3.169 \times 10^{27} \mathrm{~s}^{-1}$, $Q_{c, e x c h}=3.13 \times 10^{27} \mathrm{~s}^{-1}, l_{d, I o}=0.00125 ;\left(\right.$ dotted line) $n_{a t m o s}=10^{8} \mathrm{~cm}^{-3}$, $n_{\text {iono }}=n_{0}, Q=3.03 \times 10^{27} \mathrm{~s}^{-1}, Q_{p, \text { exch }}=5.27 \times 10^{27} \mathrm{~s}^{-1}, Q_{c, \text { exch }}=5.6 \times 10^{27} \mathrm{~s}^{-1}$, $l_{d, I o}=0.00125 ;\left(\right.$ dashed line) $n_{\text {atmos }}=5 \times 10^{8} \mathrm{~cm}^{-3}, n_{\text {iono }}=n_{0}, Q=4.04 \times 10^{27} \mathrm{~s}^{-1}$, $Q_{p, \text { exch }}=1.83 \times 10^{28} \mathrm{~s}^{-1}, Q_{c, \text { exch }}=3.97 \times 10^{28} \mathrm{~s}^{-1}, l_{d, I o}=0.0125 ;$ (dot-dashed line) $n_{\text {atmos }}=10^{9} \mathrm{~cm}^{-3}, n_{\text {iono }}=n_{0}, Q=2.1 \times 10^{27} \mathrm{~s}^{-1}, Q_{p, \text { exch }}=2.95 \times 10^{28} \mathrm{~s}^{-1}$, $Q_{c, \text { exch }}=3.75 \times 10^{28} \mathrm{~s}^{-1}, l_{d, I o}=0.0125$. 
Table 1. Dependence of torus ion charge exchange rate and pickup ion charge exchange rate versus the maximum value of atmospherical density and ionization rate $\left(Q_{0}=10^{27} \mathrm{~s}^{-1}\right)$.

* denote the regimes that fit satisfactory the observational data

\begin{tabular}{|c|c|c|c|c|c|}
\hline case & $\frac{n_{\text {atmos }}}{10^{8} \mathbf{c m}^{-3}}$ & $\frac{Q_{\text {ion }}}{Q_{0}}$ & $\frac{Q_{\mathrm{p}, \text { exch }}}{Q_{0}}$ & $\frac{Q_{c, \text { exch }}}{Q_{0}}$ & Comments \\
\hline nce & model & $\begin{array}{l}\text { without } \\
4.8\end{array}$ & $\begin{array}{l}\text { charge } \\
0\end{array}$ & $\begin{array}{l}\text { exchange } \\
0\end{array}$ & $\begin{array}{l}\text { a density is a little bit wide and it is in good agreement with an observation, } \\
\text { temperature and magnetic field are in a good agreement with an observation, Fig. 17* }\end{array}$ \\
\hline ceil & models & with & charge & exchange & $\begin{array}{l}\text { in the internal region ( } n_{\text {neutral }} \approx B \exp \frac{r-r_{10}}{H_{\text {atmos }}} \text { ) } \\
\text { density, temperature and } B_{y} \text { in satisfactory agreement with observation, Fig. 18, } \\
\text { (solid line) }\end{array}$ \\
\hline cei2 & 1 & 2.57 & & & $\begin{array}{l}\text { density and temperature in satisfactory agreement with observation, } B_{y} \text { component } \\
\text { is a weaker than observed, Fig. } 18 \text {, (dashed line) }\end{array}$ \\
\hline cei3 & 5 & 2.1 & & & $\begin{array}{l}\text { density in good agreement with observation; temperature and } B_{y} \text { are not in agreement } \\
\text { with observation }\end{array}$ \\
\hline cei4 & 10 & 1.4 & 12.6 & 14 & $\begin{array}{l}\text { density with strong narrow peak, temperature in satisfactory agreement with } \\
\text { observation; } B_{y} \text { is weaker than observed }\end{array}$ \\
\hline cei5 & 10 & 2.1 & 12 & 20.2 & $\begin{array}{l}\text { density with two peaks, temperature is in satisfactory agreement with observation; } \\
B_{y} \text { has much stronger depletion than in observation }\end{array}$ \\
\hline & models & with & charge & exchange & in the internal and external region $\left(n_{\text {neutral }} \approx \frac{A}{r^{2}}+B \exp \frac{r-r_{\text {lo }}}{H_{\text {atmos }}}\right)$ \\
\hline ceie1 & 0.5 & 3.03 & 3.169 & 3.13 & density, temperature and $B_{y}$ in good agreement with observation, Fig. $19^{*}$, (solid line) \\
\hline ceie2 & 0.5 & 4.04 & 2.67 & 3.87 & $\begin{array}{l}\text { density and temperature in satisfactory agreement with observation, } B_{y} \text { is a weaker } \\
\text { than observed }\end{array}$ \\
\hline ceie3 & 1 & 3.03 & 5.27 & 5.6 & $\begin{array}{l}\text { density is higher than the observation, variation in } B_{y} \text { is a weaker than observed, } \\
\text { Fig. 19, (dotted line) }\end{array}$ \\
\hline ceie4 & 1 & 4.04 & 5.55 & 7.93 & density is higher than observation, variation in $B_{y}$ is weaker than observed \\
\hline ceie5 & 5 & 4.04 & 18.3 & 39.7 & $\begin{array}{l}\text { density is much higher than in an observation, variation in the } B_{y} \text { is strong but profile } \\
\text { is wider than observed, Fig. } 19 \text {, (dashed line) }\end{array}$ \\
\hline ceie6 & 10 & 2.1 & 29.5 & 37.5 & $\begin{array}{l}\text { two-peak density profile, variation in the } B_{y} \text { is much stronger than observed, } \\
\text { Fig. 19, (dot-dashed line) }\end{array}$ \\
\hline ceie7 & 10 & 3.03 & 28. & 44.8 & $\begin{array}{l}\text { density is much higher than in observation, variation in the } B_{y} \text { is much weaker than } \\
\text { observed }\end{array}$ \\
\hline ceie8 & 10 & 4.04 & 27.5 & 60. & $\begin{array}{l}\text { density is much higher than in observation, variation in } B_{y} \text { is much weaker than } \\
\text { observed }\end{array}$ \\
\hline
\end{tabular}




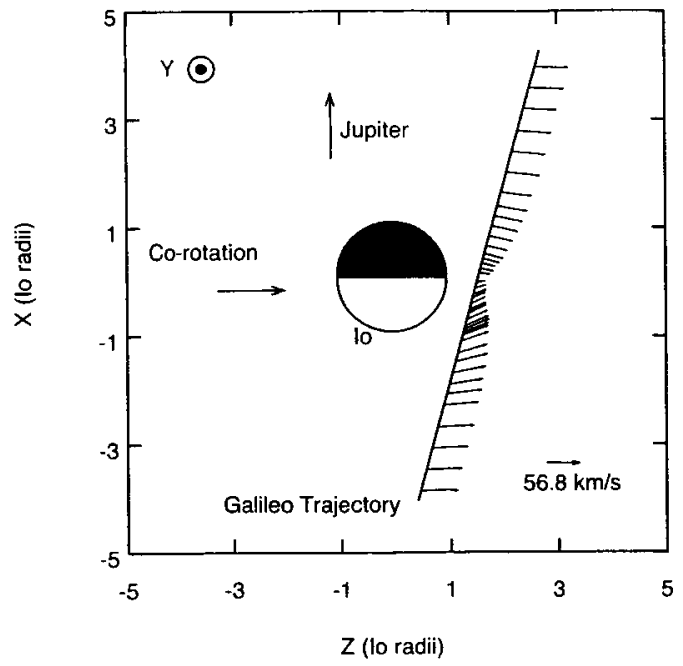

Figure 1. Galileo trajectory close to Io and the system of coordinates. 

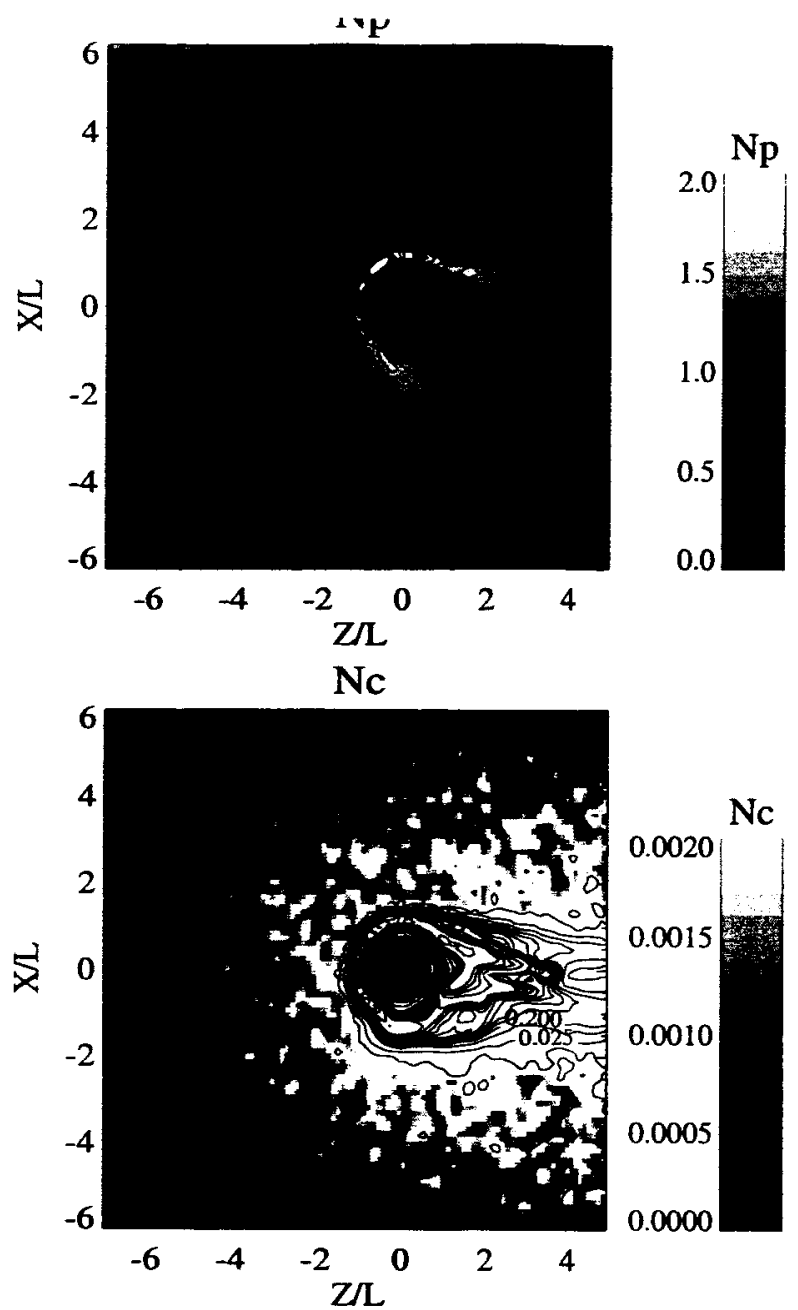

Figure 2. Incoming (top) and pickup (bottom) ion density in the $x-z$ plane. The case ceie1 (Table 1) with $\beta_{\mathrm{e}, \mathrm{PI}}=\beta_{\mathrm{e}}, \beta_{\mathrm{e}, \text { iono }}=0.25 \beta_{\mathrm{e}}, W_{\text {ext }}=5 \%$, and $H_{\text {atmos }}=0.06$. See explanation in Fig. 6 . 



Figure 3. Incoming (top) and pickup (bottom) ion density in the $y$ - $z$ plane for the same parameters as in Fig. 2. See explanation in Fig. 7. 

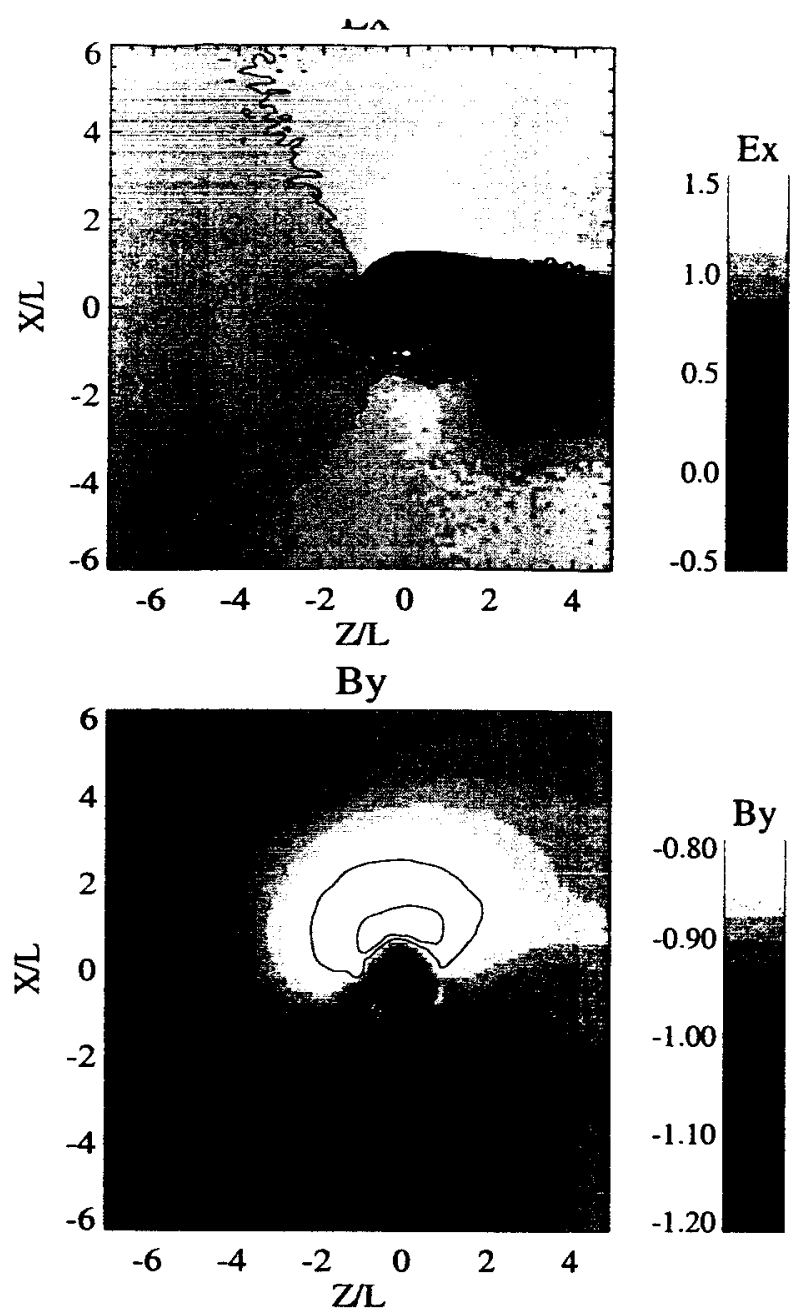

Figure 4. Electric (top) and magnetic (bottom) fields in the $x-z$ plane for the same parameters as in Fig. 2. Figure shows a strong asymmetry of the electromagnetic field due to finite gyroradius effects. 

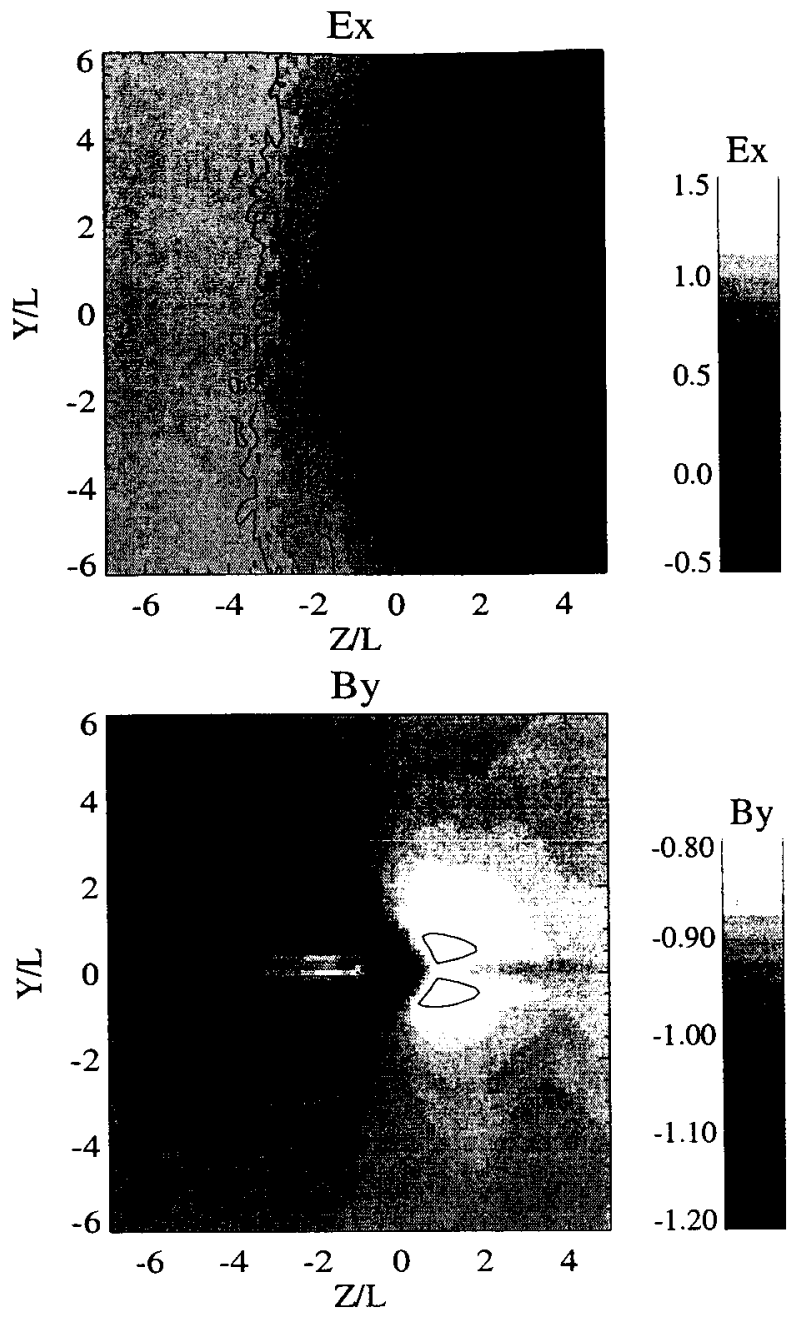

Figure 5. Electric (top) and magnetic (bottom) fields in the $y-z$ plane for the same parameters as in Fig. 2. Figure shows the formation of an Alfvén wing in the direction of the main magnetic field. 




Wc

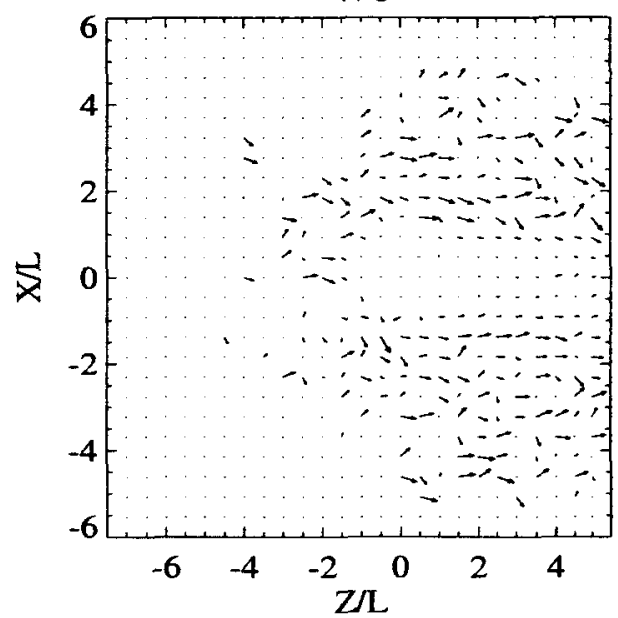

Figure 6. Incoming (top) and pickup (bottom) ion velocity arrows in the $x-z$ plane for the same parameters as in Fig. 2. The figure demonstrates a flow of pickup ions from the "corona" across the magnetic field. The incoming ions flow around the effective obstacle that is produced by pickup ions and ionosphere. 

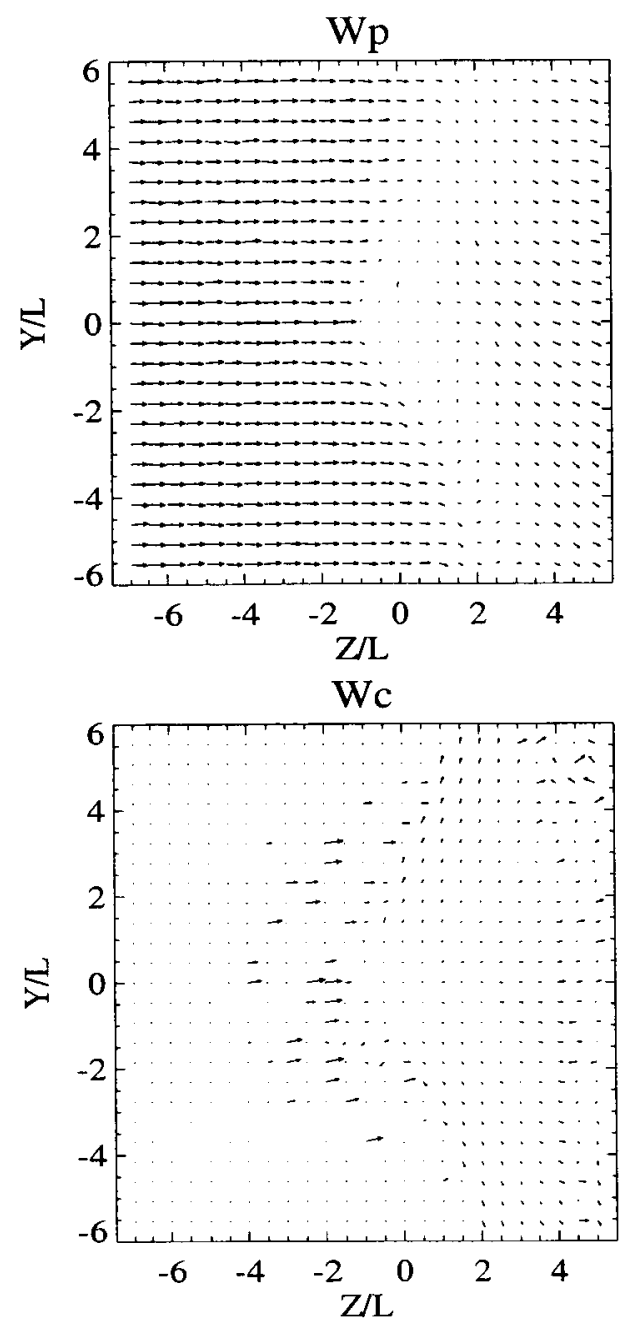

Figure 7. Incoming (top) and pickup (bottom) ion velocity arrows in the $y$ - $z$ plane for the same parameters as in Fig. 2. The figure demonstrates a strong expansion of pickup ion "corona" along the magnetic field line. The incoming ions flow around the region of extended "corona". 


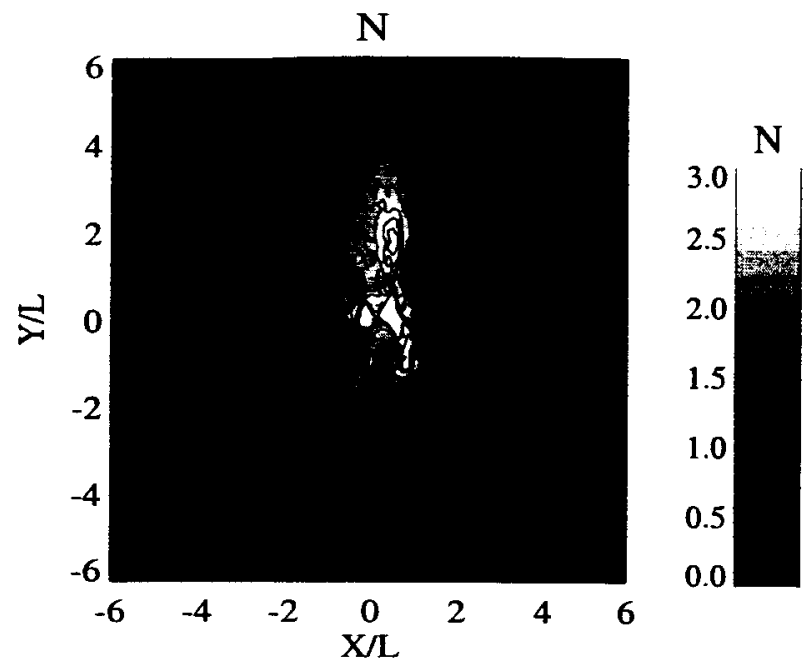

Figure 8. Two-dimensional cross section for total density in the $x-y$ plane at $z=1.5 r_{\mathrm{Io}}$ for the same parameters as in Fig. 2 . 

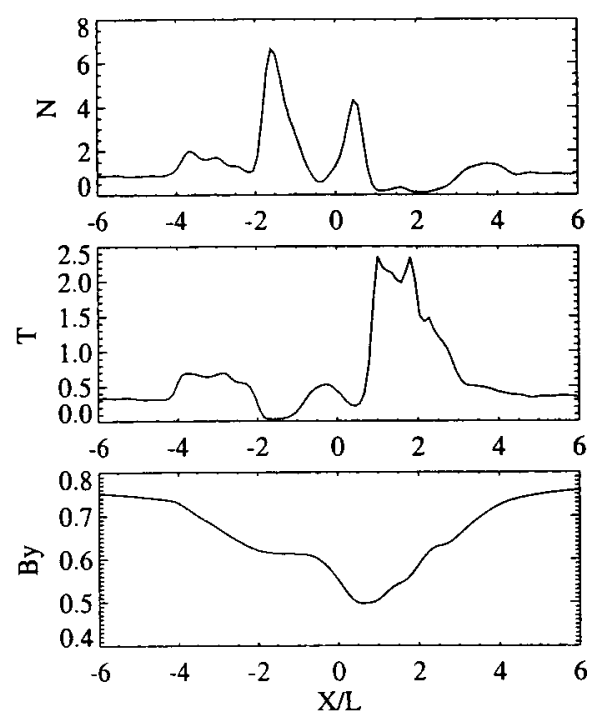

Figure 9. Total density, temperature and magnetic field along the $x$-axis at $z=1.5 r_{\text {Io }}$.

The case with $\beta_{\mathrm{e}, \mathrm{PI}}=0, \beta_{\mathrm{e} \text {,iono }}=0, W_{\text {ext }}=0 \%$, and $H_{\mathrm{atmos}}=0.06$.

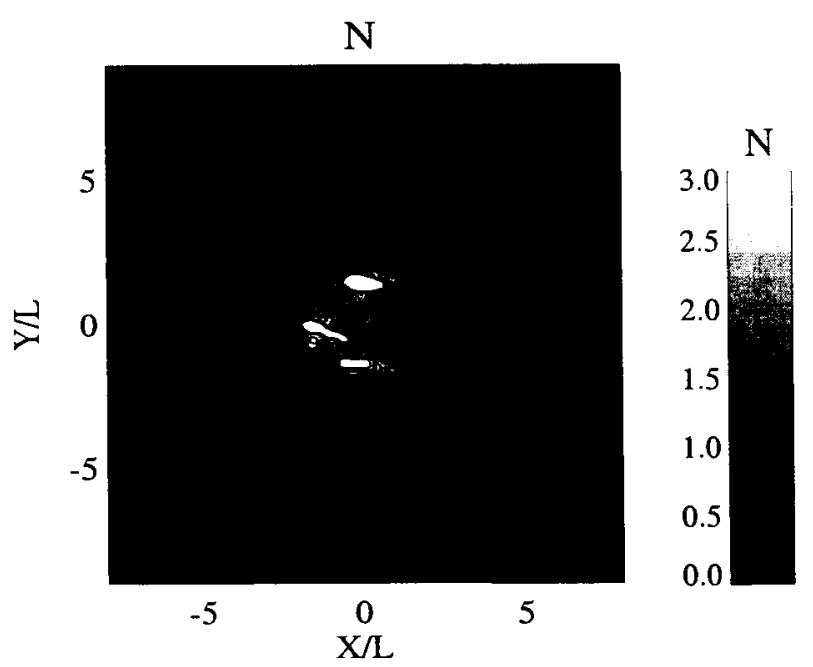

Figure 10. Two-dimensional cross section for total density in the $x$-y plane at $z=1.5 r_{\text {Io }}$.

The case with $\beta_{\mathrm{e}, \mathrm{PI}}=0, \beta_{\mathrm{e} \text {,iono }}=0, W_{\text {ext }}=0 \%$, and $H_{\text {atmos }}=0.06$. 



Figure 11. Total density, temperature and magnetic field along the $x$-axis at $z=1.5 r_{\mathrm{Io}}$.

The case with $\beta_{\mathrm{e}, \mathrm{PI}}=\beta_{\mathrm{e}}, \beta_{\mathrm{e}, \text { iono }}=0, W_{e x t}=0 \%$, and $H_{\text {atmos }}=0.06$.

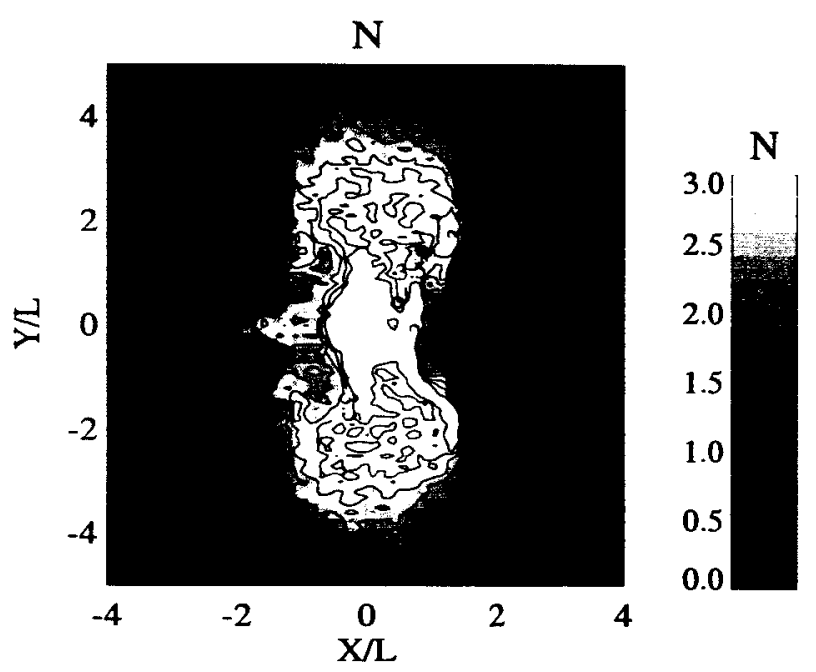

Figure 12. Two-dimensional cross section for total density in the $x-y$ plane at $z=1.5 r_{\mathrm{I}}$.

The case with $\beta_{\mathrm{e}, \mathrm{PI}}=\beta_{\mathrm{e}}, \beta_{\mathrm{e}, \text { iono }}=0, W_{\text {ext }}=0 \%$, and $H_{\text {atmos }}=0.06$. 

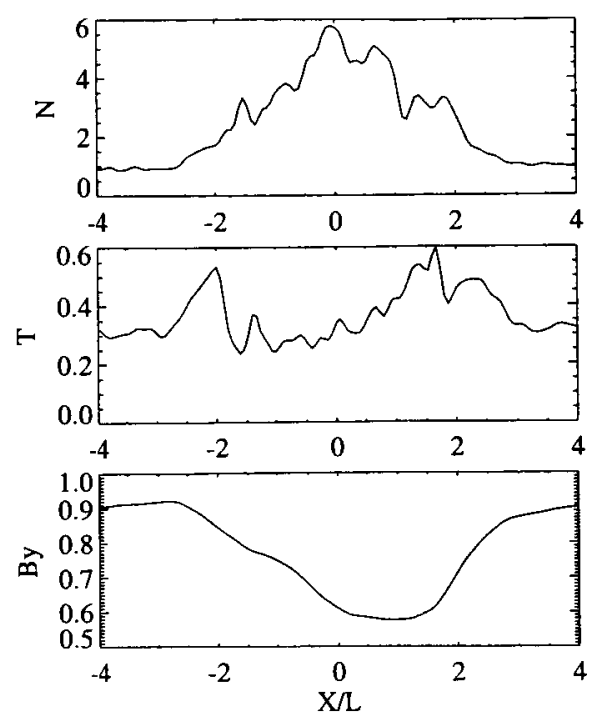

Figure 13. Total density, temperature and magnetic field along the $x$-axis at $z=1.5 r_{\mathrm{Io}}$.

The case with $\beta_{\mathrm{e}, \mathrm{PI}}=\beta_{\mathrm{e}}, \beta_{\mathrm{e}, \text { iono }}=\beta_{\mathrm{e}}, W_{\text {ext }}=0 \%, H_{\mathrm{atmos}}=0.06$.

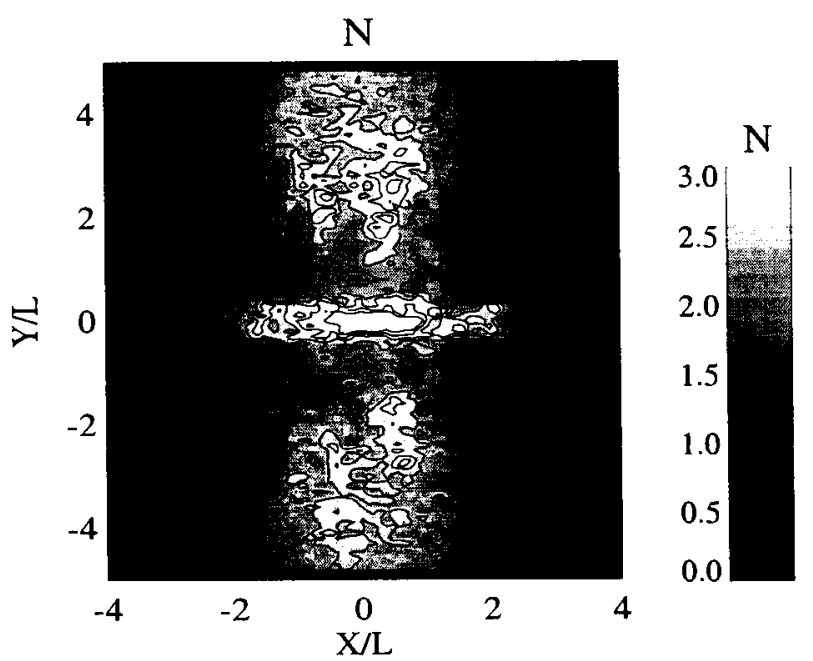

Figure 14. Two-dimensional cross section for total density in the $x$-y plane at $z=1.5 r_{\mathrm{Io}}$.

The case with $\beta_{\mathrm{e}, \mathrm{PI}}=\beta_{e}, \beta_{\mathrm{e}, \text { iono }}=\beta_{\mathrm{e}}, W_{\text {ext }}=0 \%, H_{\mathrm{atmos}}=0.06$. 


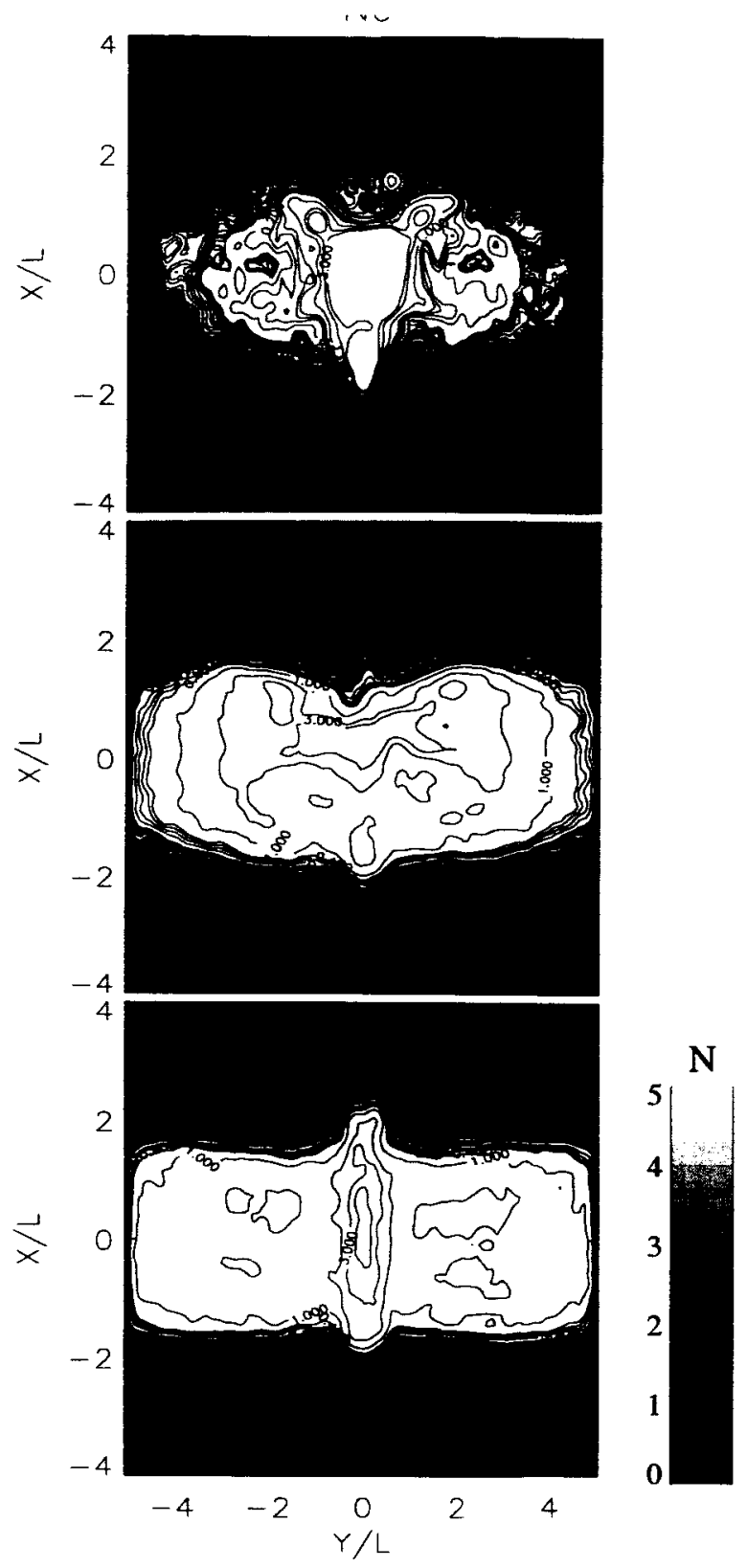

Figure 15. Two-dimensional cross sections for pickup ion density in the $x-y$ plane at $z=1.5 r_{\mathrm{Io}}$. The cases with (top) $\beta_{\mathrm{e}, \mathrm{PI}}=0, \beta_{\mathrm{e}, \text { iono }}=0 ;$ (middle) $\beta_{\mathrm{e}, \mathrm{PI}}=\beta_{\mathrm{e}}, \beta_{\mathrm{e}, \text { iono }}=0$; (bottom) $\beta_{\mathrm{e}, \mathrm{PI}}=\beta_{e}, \beta_{\mathrm{e}, \mathrm{iono}}=0.25 \beta_{e} . W_{e x t}=0 \%$, and $H_{\mathrm{atmos}}=0.06$. Figure demonstrates asymmetry of pickup ion density across the wake. 


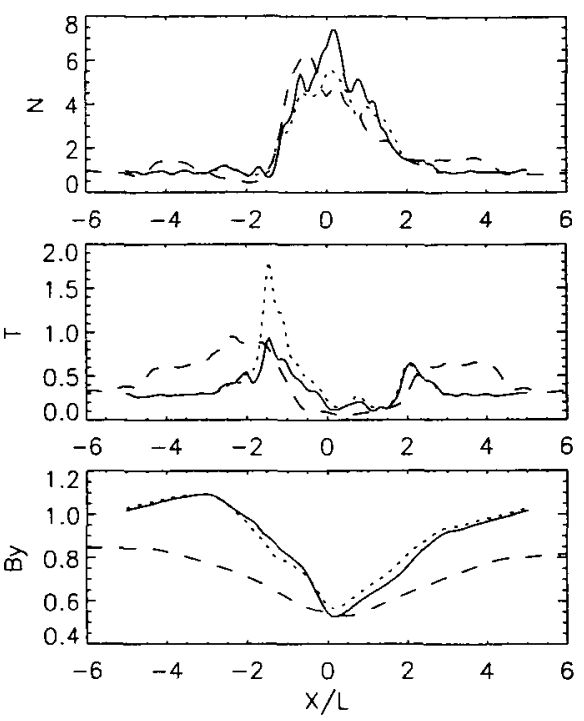

Figure 16. Total density, temperature and magnetic field along the $x$-axis at $z=1.5 r_{\text {Io }}$ for cases with $\beta_{\mathrm{e}, \mathrm{PI}}=\beta_{\mathrm{e}}, \beta_{\mathrm{e}, \text { iono }}=0.25 \beta_{\mathrm{e}}, H_{\mathrm{atmos}}=0.06, l_{\mathrm{d}, \mathrm{up}}=0.0025$. (solid line) $W_{\text {ext }}=10 \%, l_{\mathrm{d}, \text { iono }}=0.0025 ;($ dotted line $) W_{\text {ext }}=30 \%, l_{\mathrm{d}, \text { iono }}=0.0025 ;($ dashed line $)$ $W_{\text {ext }}=10 \%, l_{\mathrm{d}, \mathrm{iono}}=0.025$. 

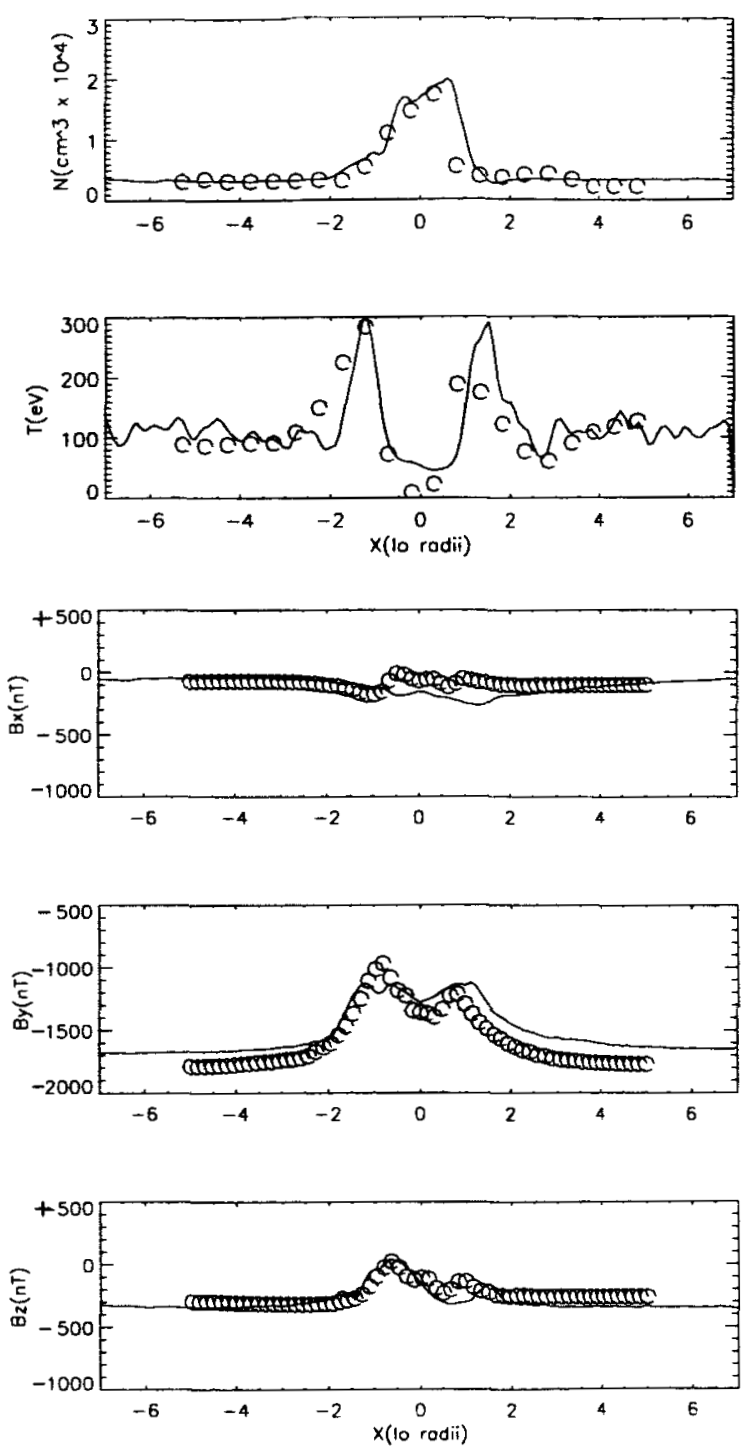

Figure 17. Comparison of Galileo PLS (I0 pass) [Frank et al., 1996] and MAG [Kivelson et al., 1996] (the open circles) data with the Io hybrid model results along the trajectory in the absence of charge exchange (case nce, Table 1). $\beta_{\mathbf{e}, \mathrm{PI}}=0.125 \beta_{\mathbf{e}}$, $\beta_{\mathrm{e}, \text { iono }}=0.125 \beta_{\mathrm{e}}, W_{\text {ext }}=5 \%, H_{\mathrm{atmos}}=0.06$, and $n_{\mathrm{iono}}=800(100) n_{0} . l_{\mathrm{d}, \mathrm{up}}=0.00125$ and $l_{\mathrm{d}, \mathrm{Io}}=0.0125$. 



Figure 18. Same as Fig. 17, but with charge exchange $\left(B \exp \frac{r-r_{\mathrm{b}}}{H_{\text {atmos }}}\right), n_{\text {atmos }}=10^{8} \mathrm{~cm}^{-3}$ : (solid line, case cei1, Table 1); (dashed line, case cei2, Table 1). 




Figure 19. Same as Fig. 17, but with charge exchange $\left(\frac{A}{r^{2}}+B \exp \frac{r-r_{l}}{H_{\text {atmos }}}\right)$ : (solid line, case ceie1, Table 1) $n_{\text {iono }}=n_{0}, l_{\mathrm{d}, \text { Io }}=0.00125 ;($ dotted line, case ceie 3 , Table 1$) l_{\mathrm{d}, \mathrm{Io}}=0.00125$ (dashed line, case ceie5, Table 1) $l_{\mathrm{d}, \mathrm{Io}}=0.0125 ;$ (dot-dashed line, case ceie6, Table 1) $l_{\mathrm{d}, \mathrm{Io}}=0.0125$. 